\title{
Dynamical Analysis, Synchronization, Circuit Design, and Secure Communication of a Novel Hyperchaotic System
}

\author{
Li Xiong, ${ }^{1,2}$ Zhenlai Liu, $^{1}$ and Xinguo Zhang ${ }^{3}$ \\ ${ }^{1}$ School of Physics and Electromechanical Engineering, Hexi University, Zhangye 734000, China \\ ${ }^{2}$ State Key Laboratory of ASIC \& System, Fudan University, Shanghai 200433, China \\ ${ }^{3}$ School of Information Science and Engineering, Lanzhou University, Lanzhou 730000, China
}

Correspondence should be addressed to Li Xiong; xl-427814@163.com

Received 19 May 2017; Revised 22 August 2017; Accepted 26 September 2017; Published 15 November 2017

Academic Editor: Amr Elsonbaty

Copyright (C) 2017 Li Xiong et al. This is an open access article distributed under the Creative Commons Attribution License, which permits unrestricted use, distribution, and reproduction in any medium, provided the original work is properly cited.

\begin{abstract}
This paper is devoted to introduce a novel fourth-order hyperchaotic system. The hyperchaotic system is constructed by adding a linear feedback control level based on a modified Lorenz-like chaotic circuit with reduced number of amplifiers. The local dynamical entities, such as the basic dynamical behavior, the divergence, the eigenvalue, and the Lyapunov exponents of the new hyperchaotic system, are all investigated analytically and numerically. Then, an active control method is derived to achieve global chaotic synchronization of the novel hyperchaotic system through making the synchronization error system asymptotically stable at the origin based on Lyapunov stability theory. Next, the proposed novel hyperchaotic system is applied to construct another new hyperchaotic system with circuit deformation and design a new hyperchaotic secure communication circuit. Furthermore, the implementation of two novel electronic circuits of the proposed hyperchaotic systems is presented, examined, and realized using physical components. A good qualitative agreement is shown between the simulations and the experimental results around $500 \mathrm{kHz}$ and below $1 \mathrm{MHz}$.
\end{abstract}

\section{Introduction}

The Lorenz chaotic system was proposed [1] and later the chaotic synchronization was implemented in the electronic circuit [2], which greatly inspired many scientists and accelerated the pace of chaos research [3-7]. A hyperchaotic system is defined as an attractor with at least two positive Lyapunov exponents and an autonomous system with phase space of dimension at least four [8]. The sum of Lyapunov exponents must be negative to ensure that the system is dissipative [9]. The hyperchaotic systems have more complex structure, higher unpredictability, and more randomness than ordinary chaotic systems. Thus, the hyperchaotic attractors are more suitable for many important fields in applied nonlinear sciences such as secure communications, neural network, image encryption, laser physics, and nonlinear circuits [1019].

With the deep development of chaotic systems, synchronization of hyperchaotic systems is an important nonlinear phenomenon. In recent years, Mahmoud et al. introduced some chaotic and hyperchaotic systems with complex variables, analyzed their chaotic behavior, and proposed several types of synchronization methods [20-25]. Usually, increasingly novel chaotic systems are generated from low-order chaotic systems to hyperchaotic systems [26-28] and from two-wing systems to four-wing or multiloop systems [29-32]. A significant application of chaotic systems is to form chaotic secure communication circuit [33]. From the perspective of improving the security of secure communication system, the novel hyperchaotic system should be selected. Accordingly, most hyperchaotic systems are generated and verified by numerical simulations; there is a certain degree of deviation from the physical circuit system [34]. Therefore, hardware circuit simulation and physical verification are also important for hyperchaotic system generation.

The contribution of this paper is that we introduce a novel fourth-order hyperchaotic system on the basis of a modified Lorenz-like chaotic circuit with reduced number of 
amplifiers. Then we perform a detailed qualitative analysis, active control, synchronous stability analysis, and applications of the novel hyperchaotic system. The active control and synchronization results derived in this paper are established by using Lyapunov stability theory. The corresponding physical circuit design for the novel hyperchaotic system is also proposed to show the accuracy and efficiency of circuit realization. The analog circuit implementation results match the Multisim and Matlab simulation results. These proposed circuit design methods can also be applied in other complex hyperchaotic systems.

This paper is organized as follows. In Section 2, a modified Lorenz-like chaotic circuit is constructed with reduced number of amplifiers. Based on the modified Lorenz-like chaotic circuit, several qualitative issues about a novel hyperchaotic system, such as the basic dynamical behavior, divergence, equilibria, Lyapunov exponents, and synchronous stability, are investigated analytically and numerically in Section 3. In Section 4 , another $z-u-y$ hyperchaotic system and a new hyperchaotic secure communication circuit are proposed based on the novel $(y+z)-u-x$ hyperchaotic circuit. In Section 5, the proposed hyperchaotic circuits are, respectively, implemented in analog electronic circuits. Finally, some conclusions and discussions are drawn in Section 6.

\section{Circuit Design of a Modified Lorenz-Like Chaotic System}

A modified Lorenz-like chaotic system is proposed by the following autonomous nonlinear system of differential equations:

$$
\begin{aligned}
& \dot{x}=a(y-x) \\
& \dot{y}=b x-y+c x z \\
& \dot{z}=-d x y-e z,
\end{aligned}
$$

where $x, y$, and $z$ are the state variables and $a, b, c, d$, and $e$ are constant, positive parameters of the system. When choosing $a=10, b=45, c=20, d=5$, and $e=3.7$, there exists a typical chaotic attractor in system (1).

$$
\begin{aligned}
& \dot{x}=-10 x+10 y \\
& \dot{y}=45 x-y+20 x z \\
& \dot{z}=-5 x y-3.7 z .
\end{aligned}
$$

The two- and three-dimensional chaotic attractors with Matlab simulation of the modified Lorenz-like chaotic system (2) are shown in Figure 1. It can be seen from the numerical simulation results that the numerical range of each variable parameter is within $-10 \mathrm{~V}$ to $+10 \mathrm{~V}$, and it fully meets the requirements of circuit design in practical applications. That is because the working voltage range of electronic components is generally from $-15 \mathrm{~V}$ to $+15 \mathrm{~V}$ in practical electronic circuits. Therefore, it must be the equation of scaling if the circuit is to be implemented.
Based on the modified Lorenz-like chaotic system (2), the normalized resistor is set as $R=100 \mathrm{k} \Omega$ in order to design the Lorenz-like chaotic circuit. Thus, the state equation of the Lorenz-like chaotic circuit is obtained as follows:

$$
\begin{aligned}
& \dot{x}=-\frac{100 \mathrm{k}}{10 \mathrm{k}} x+\frac{100 \mathrm{k}}{10 \mathrm{k}} y \\
& \dot{y}=\frac{100 \mathrm{k}}{2.2 \mathrm{k}} x-\frac{100 \mathrm{k}}{100 \mathrm{k}} y+\frac{100 \mathrm{k}}{0.51 \mathrm{k}} \times 0.1 x z \\
& \dot{z}=-\frac{100 \mathrm{k}}{2 \mathrm{k}} \times 0.1 x y-\frac{100 \mathrm{k}}{27 \mathrm{k}} z .
\end{aligned}
$$

Therefore, the Lorenz-like chaotic circuit schematic is designed as shown in Figure 2. It can be seen from Figure 2 that the circuit is composed of five operational amplifiers and two analog multipliers. It can output three waveforms and three phase portraits, and it can output stable third-order double vortex chaotic signal. Being different from the general Lorenz-like circuit, it can output inverted phase portrait shape like butterfly wings. However, lacking of improvement on the Lorenz-like chaotic circuit precluded comprehensive statements. Although it can implement the function of (2), it is not the most optimal circuit. In order to obtain the most optimal circuit, the Lorenz-like chaotic circuit shown in Figure 2 needs to be improved. The basic idea of improved design is to simplify, minimize, and merge the circuit by using the main knowledge of circuit theory under the condition of not changing the circuit function. Thus, an improved Lorenzlike chaotic circuit consisting of four operational amplifiers and two analog multipliers is designed based on Figure 2.

The final improved design result of the Lorenz-like circuit is shown in Figure 3. It consists of 4 operational amplifiers, 2 analog multipliers, 9 resistors, and 3 capacitors. As can be seen from Figure 3, the number of operational amplifiers is reduced from 5 to 4 , and other passive components are correspondingly reduced. Through improvement, the complexity of the circuit, the thermal noise of the total resistance, and the total error of the circuit can also be reduced. Meanwhile, the cost can be reduced. Thus, the improved Lorenz-like chaotic circuit is simple, easy to debug, and suitable for mass production. From this, the various curves of chaos evolution of the improved Lorenz-like chaotic circuit can be observed.

The beneficial effects of the improved Lorenz-like circuit are as follows: (i) It can output three chaotic waveform signals of $x, y$, and $z$ and three chaotic phase portraits of $x y, x z$, and $z y$. (ii) A variety of chaotic signals can be displayed on the oscilloscope. (iii) It can output inverted phase portrait shape like butterfly wings and other kinds of experiments can also be carried out by the improved circuit. Using these values, some simulations are implemented. All of the electronic components are easily available. The chaotic phase portraits of the improved Lorenz-like chaotic circuit by Multisim are shown in Figure 4. It can be seen from the Multisim simulation results that it is consistent with the Matlab simulation results shown in Figure 1. That is, it fully conforms to the requirements of the circuit design in practical applications. 


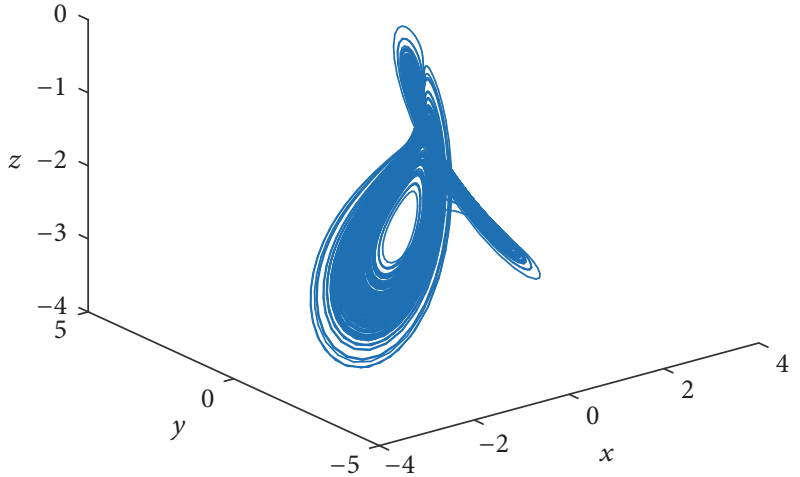

(a) $x y z$ plane

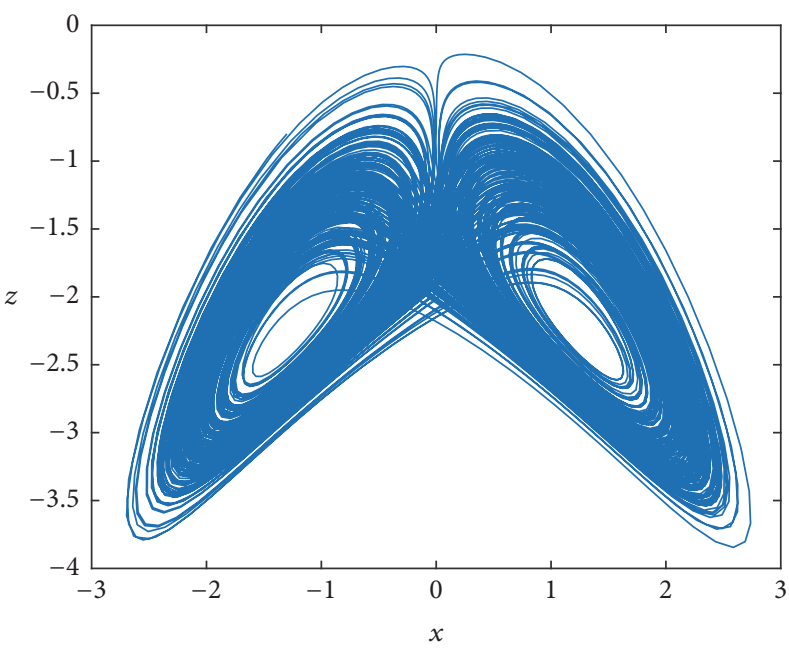

(c) $x z$ plane

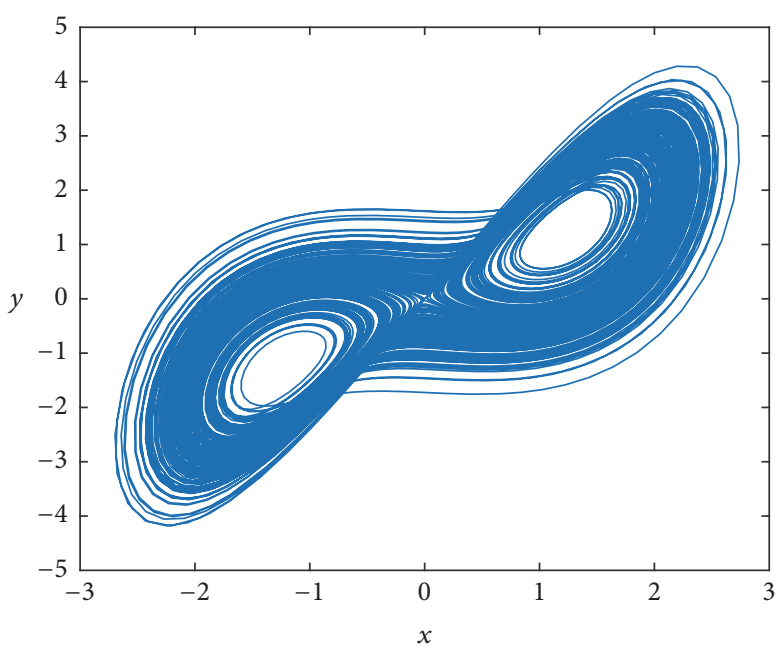

(b) $x y$ plane

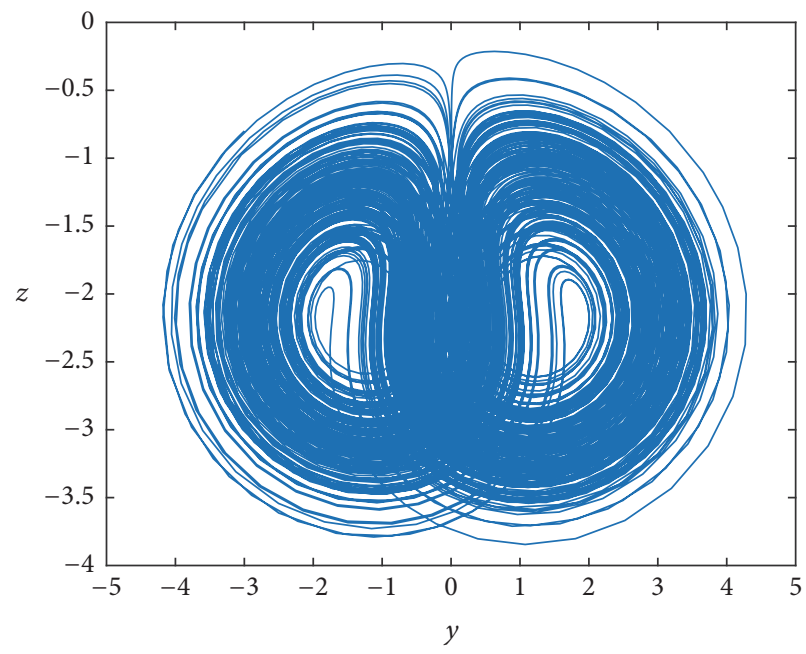

(d) $z y$ plane

Figure 1: The chaotic attractors of the modified Lorenz-like chaotic system.

\section{Dynamical Analysis of the Novel Hyperchaotic System Based on the Improved Lorenz-Like Chaotic Circuit}

It can be seen from the analysis and simulations above that the improved Lorenz-like chaotic circuit can only output three chaotic waveforms and three chaotic phase portraits. What is more, it can only output inverted phase portrait shape like butterfly wings. However, in order to meet certain conditions of displaying four chaotic waveforms, six chaotic phase portraits, and upright phase portrait shape like butterfly wings, the improved Lorenz-like chaotic circuit as shown in Figure 3 needs to be deformed from low-order chaotic system to hyperchaotic system.

3.1. Divergence and Equilibria of the Novel Hyperchaotic System. On the basis of the improved Lorenz-like chaotic circuit shown in Figure 3, the input is introduced by $(y+z)$ end, and feedback is given to the $x$ input stage after the $u$ stage. Thus, a novel fourth-order hyperchaotic circuit is constructed and the proposed hyperchaotic circuit schematic is shown in Figure 5. It is composed of 5 operational amplifiers, 2 analog multipliers, 12 resistors, and 4 capacitors. In order to facilitate the narrative, we call this novel circuit $(y+z)-u-x$ hyperchaotic circuit.

The beneficial effects of the novel $(y+z)-u-x$ fourthorder hyperchaotic circuit are as follows: it not only can output four chaotic waveforms and six phase portraits, but also can output stable fourth-order double vortex chaotic signals. Also, operational amplifier TL082 or TL084 and analog multiplier AD633 are used. According to the new $(y+z)-u-x$ hyperchaotic circuit, the normalized resistor is set as $R=100 \mathrm{k} \Omega$. Thus, the state equation of the corresponding hyperchaotic circuit is obtained as follows:

$$
\begin{aligned}
& \dot{x}=-\frac{100 \mathrm{k}}{100 \mathrm{k}} x-\frac{100 \mathrm{k}}{100 \mathrm{k}} y-\frac{100 \mathrm{k}}{100 \mathrm{k}} \times 0.1 u \\
& \dot{y}=-\frac{100 \mathrm{k}}{22 \mathrm{k}} x+\frac{100 \mathrm{k}}{510 \Omega} \times 0.01 x z
\end{aligned}
$$



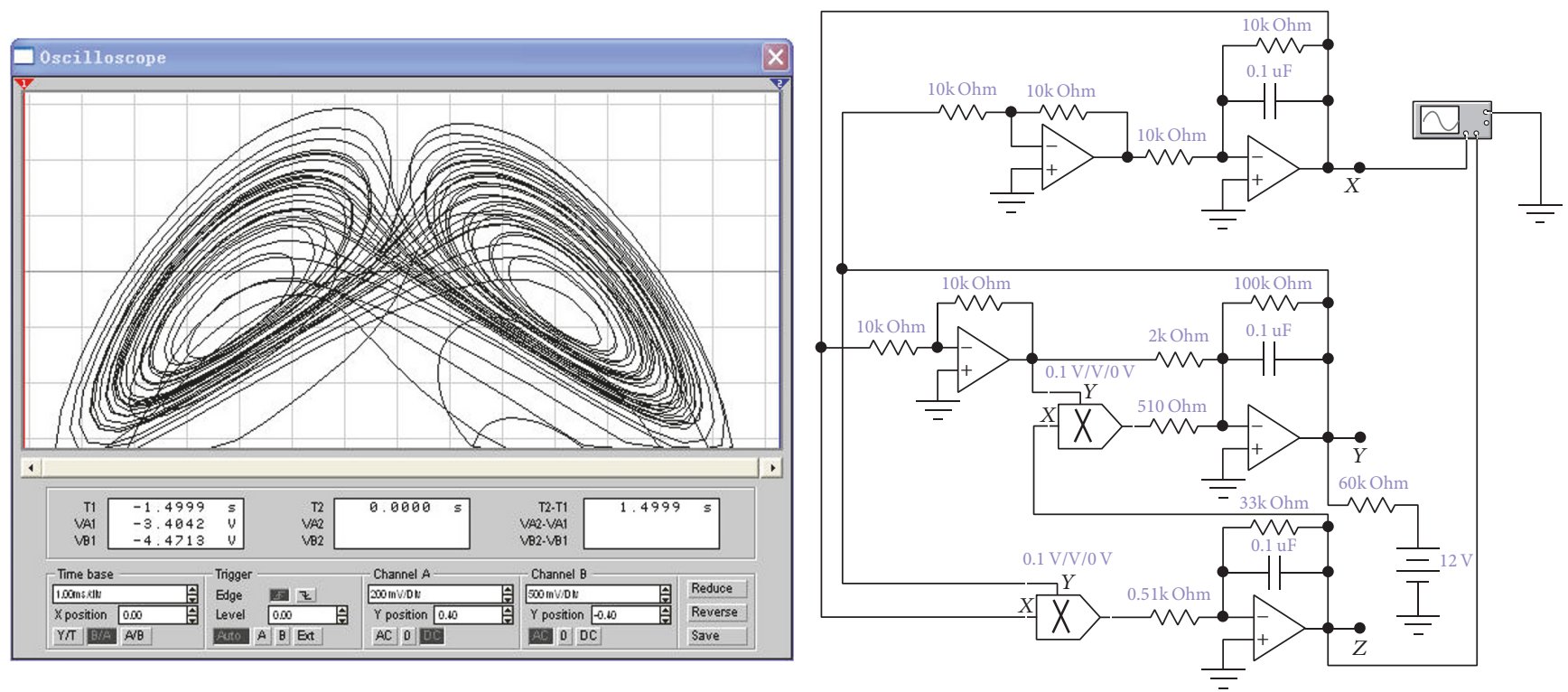

FIGURE 2: Lorenz-like chaotic circuit of system (2) using 5 amplifiers.

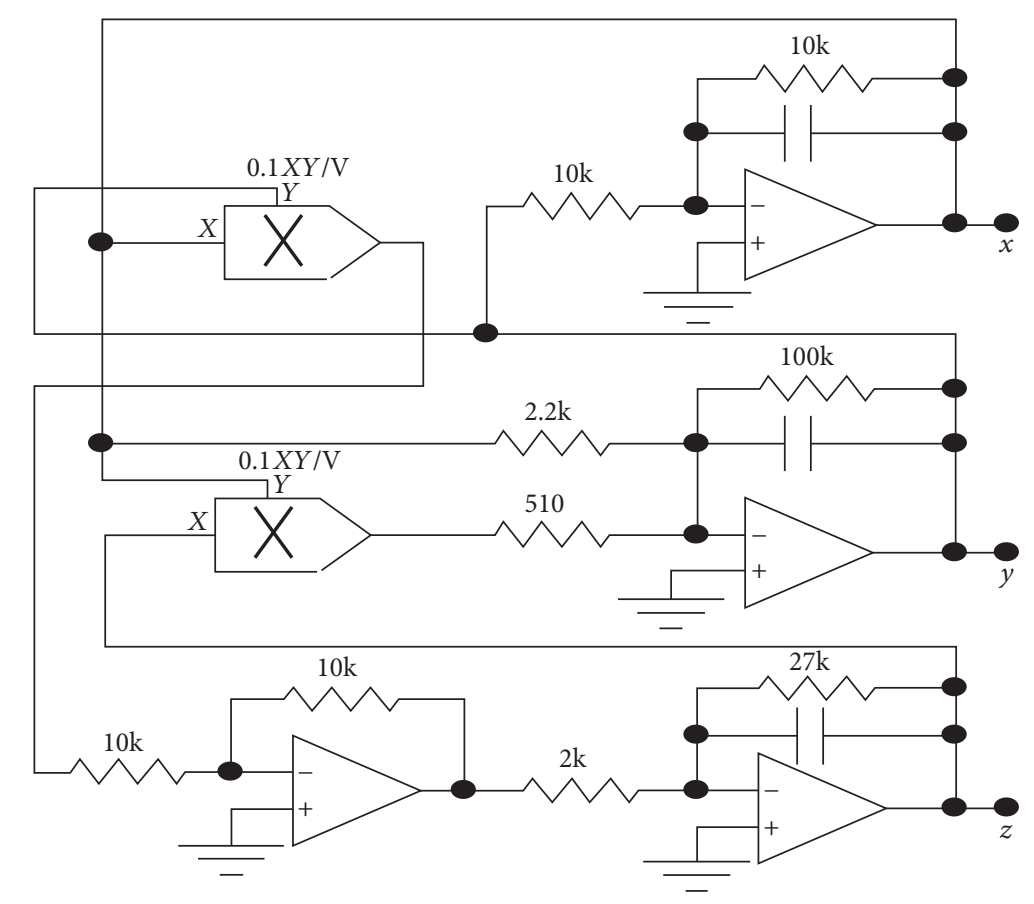

FIGURE 3: Lorenz-like chaotic circuit of system (2) with reduced number of amplifiers.

$$
\begin{aligned}
& \dot{z}=-\frac{100 \mathrm{k}}{270 \mathrm{k}} z-\frac{100 \mathrm{k}}{2 \mathrm{k}} \times 0.01 x y \\
& \dot{u}=-\frac{100 \mathrm{k}}{100 \mathrm{k}} y-\frac{100 \mathrm{k}}{100 \mathrm{k}} z-\frac{100 \mathrm{k}}{20 \mathrm{k}} \times 0.1 u .
\end{aligned}
$$

Then, a novel fourth-order hyperchaotic system is obtained by (4):

$$
\begin{aligned}
& \dot{x}=-x-y-a u \\
& \dot{y}=-b x+c x z
\end{aligned}
$$




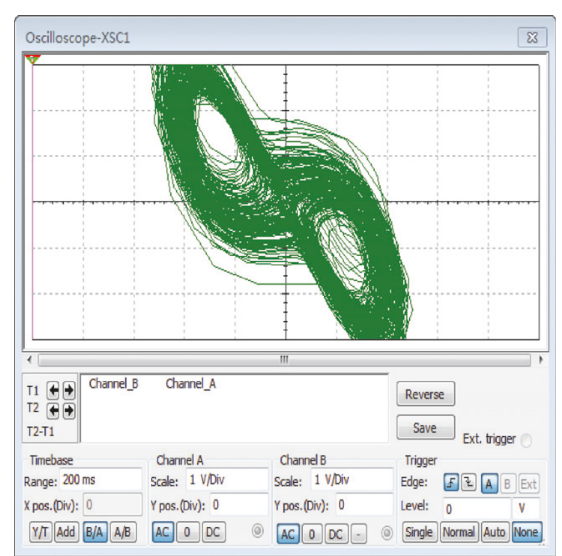

(a) $x y$ phase portrait

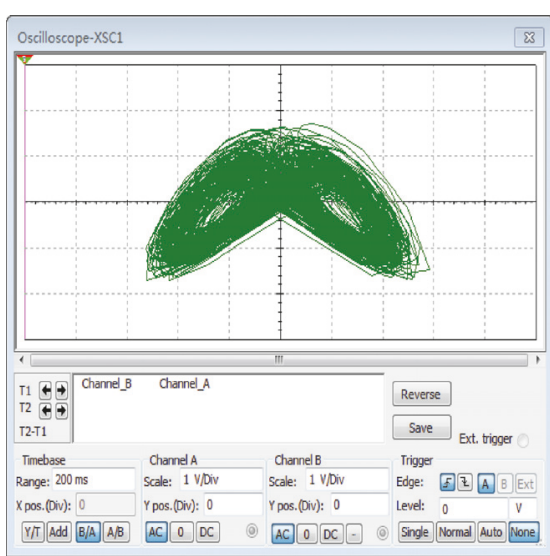

(b) $x z$ phase portrait

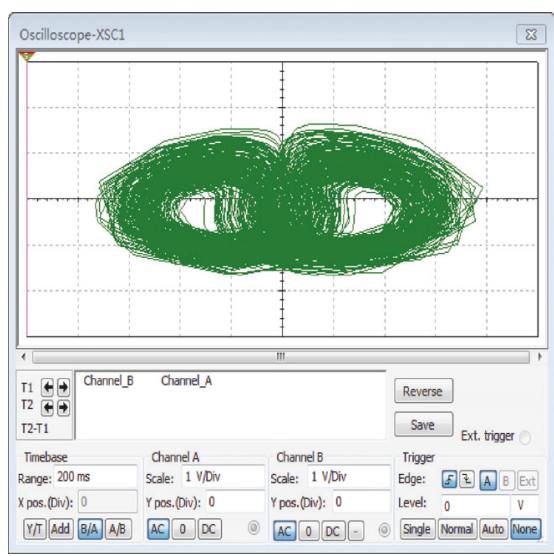

(c) $z y$ phase portrait

FIGURE 4: Chaotic phase portraits of the Lorenz-like chaotic circuit with reduced number of amplifiers.

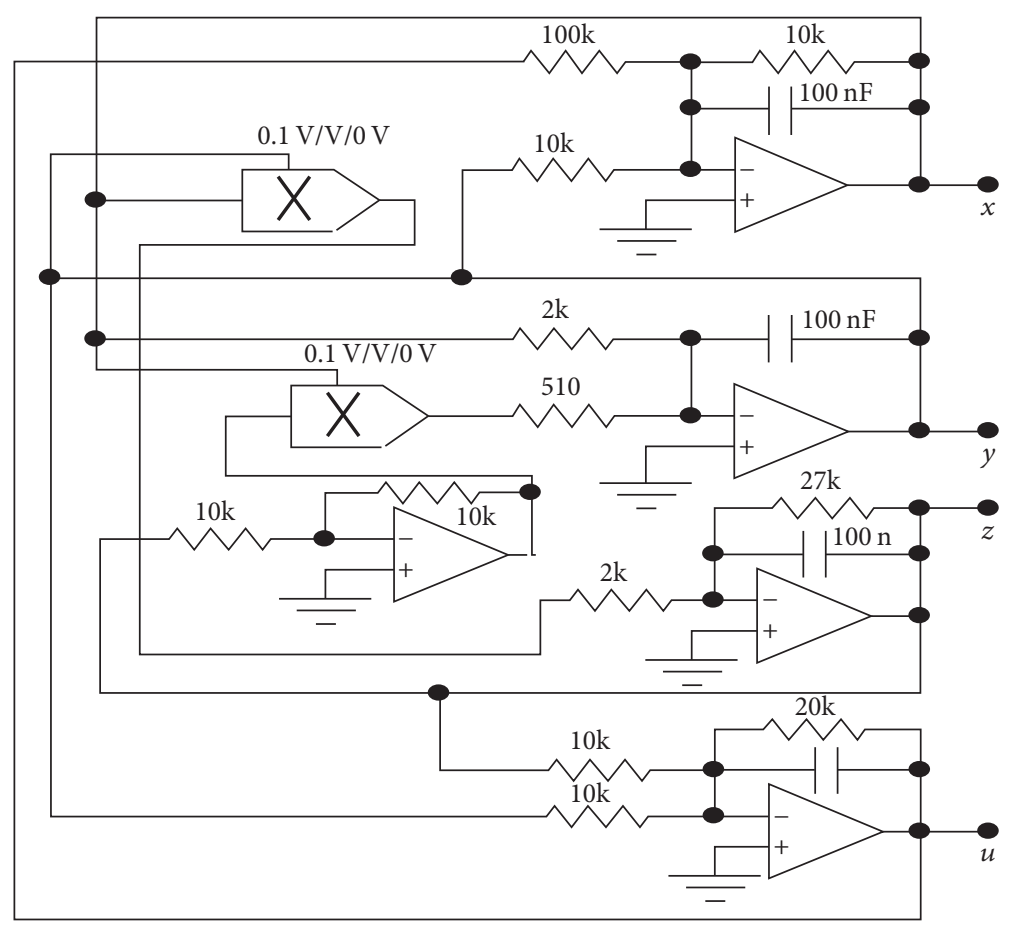

Figure 5: $(y+z)-u-x$ hyperchaotic circuit schematic.

$$
\begin{aligned}
p t] \dot{z} & =-d z-e x y \\
\dot{u} & =-y-z-e u
\end{aligned}
$$

where $x, y, z$, and $u$ are the state variables and $a, b, c, d$, and $e$ are constant, positive parameters of the system. When choosing $a=0.1, b=4.55, c=1.96, d=0.37$, and $e=$ 0.5 , the novel system (5) is chaotic. Substituting the specific parameter values, (5) becomes

$$
\begin{aligned}
& \dot{x}=-x-y-0.1 u \\
& \dot{y}=-4.55 x+1.96 x z
\end{aligned}
$$

$$
\begin{aligned}
& \dot{z}=-0.37 z-0.5 x y \\
& \dot{u}=-y-z-0.5 u
\end{aligned}
$$

This is the novel fourth-order hyperchaotic system. The divergence of the hyperchaotic system (5) is easily calculated as

$\nabla=\frac{\partial \dot{x}}{\partial x}+\frac{\partial \dot{y}}{\partial y}+\frac{\partial \dot{z}}{\partial z}+\frac{\partial \dot{u}}{\partial u}=-1-d-e=-1.87<0$.

The divergence is less than 0 . A necessary and sufficient condition for system (5) to be dissipative is that the divergence of the vector field is negative when the time tends to be 
infinite, and the corresponding dynamic characteristics will be presented.

Consider $\dot{x}=\dot{y}=\dot{z}=\dot{u}=0$; then the equilibrium equation of system (5) is easily obtained as follows:

$$
\begin{aligned}
& \dot{x}=-x-y-a u=0 \\
& \dot{y}=-b x+c x z=0 \\
& \dot{z}=-d z-e x y=0 \\
& \dot{u}=-y-z-e u=0 .
\end{aligned}
$$

Clearly, the solution of (8) is the origin; that is, the equilibrium point of system (5) is obtained as

$$
\begin{aligned}
& x=0, \\
& y=0, \\
& z=0, \\
& u=0 .
\end{aligned}
$$

The Jacobian matrix for system (5) at equilibrium point (9) is obtained as

$$
J=\left[\begin{array}{cccc}
-1 & -1 & 0 & -a \\
-b+c z & 0 & c x & 0 \\
-e y & -e x & -d & 0 \\
0 & -1 & -1 & -e
\end{array}\right]
$$

where $a=0.1, b=4.55, c=1.96, d=0.37$, and $e=0.5$. Then, the Jacobian matrix for system (5) at equilibrium point is easily obtained as

$$
J=\left[\begin{array}{cccc}
-1 & -1 & 0 & -0.1 \\
-4.55+1.96 z & 0 & 1.96 x & 0 \\
-0.5 y & -0.5 x & -0.37 & 0 \\
0 & -1 & -1 & -0.5
\end{array}\right]
$$

The characteristic polynomial of the Jacobian matrix (11) is described as follows:

$$
\operatorname{det}(\lambda I-J)=0 .
$$

Therefore, the solutions of (12) are obtained as $\lambda_{1}=$ $-2.7368, \lambda_{2}=1.6419, \lambda_{3}=-0.4050$, and $\lambda_{4}=0.3700$. Because there are two positive values in the four eigenvalues above, the equilibrium point $\{x=y=z=u=0\}$ is an unstable equilibrium point. It shows that the novel fourthorder hyperchaotic system is unstable in two directions and stable in the other two directions.

3.2. Chaotic Attractors and Lyapunov Exponents. All of the electronic components are easily available. The hyperchaotic phase portraits of the new hyperchaotic circuit by Multisim are shown in Figure 6. And the hyperchaotic phase portraits by Matlab are shown in Figure 7. It can be seen from the simulation results that it can output six chaotic phase portraits of $x y, x z, z y, x u, y u$, and $z u$. More importantly, being different from the modified Lorenz-like circuit, it can output upright phase portrait shape like butterfly wings. Moreover, it can be seen from the numerical simulation results that the numerical range of each variable parameter is within $-10 \mathrm{~V}$ to $+10 \mathrm{~V}$, and it is consistent with the Multisim and Matlab simulation results shown in Figures 6 and 7. That is, it fully conforms to the requirements of circuit design in practical applications. A variety of hyperchaotic signals can be displayed on the oscilloscope.

In order to further verify the chaotic dynamical behavior of the novel hyperchaotic system (5), the Lyapunov exponents of the novel hyperchaotic system are determined numerically. With the parameters chosen as $a=0.1, b=4.55, c=1.96, d=$ 0.37 , and $e=0.5$, the corresponding Lyapunov exponents are obtained as follows: $L_{1}=0.0717, L_{2}=0.0209, L_{3}=-0.4187$, and $L_{4}=-1.5439$. Thus, the Lyapunov dimension $[8,21]$ of the new hyperchaotic system (5) is also calculated as

$$
D_{L}=M+\frac{1}{\left|L_{M+1}\right|} \sum_{i=1}^{M} L_{i}
$$

such that $M$ is the largest integer, for which

$$
\begin{aligned}
& \sum_{i=1}^{M} L_{i}>0, \\
& \sum_{i=1}^{M+1} L_{i}<0 .
\end{aligned}
$$

This means that system (5) is a hyperchaotic system since $L_{1}$ and $L_{2}$ are positive values and a dissipative system since the sum of the Lyapunov exponents is negative. It meets the condition of at least two positive Lyapunov exponents for the hyperchaotic systems. The Lyapunov dimension of this hyperchaotic attractor using (13) and (14) is $D_{L}=$ 2.2211. Therefore, we conclude that the Lyapunov dimension of the new hyperchaotic system (5) is fractional. And the corresponding Lyapunov exponents diagram of the novel hyperchaotic system (6) is shown in Figure 8.

3.3. Synchronous Stability Analysis. From a physical point of view, synchronization means that the trajectory of a system converges to another system and maintains a consistent dynamic phenomenon. Consider two chaotic systems, and one of the chaotic systems is described as

$$
\dot{X}=F(X, t) \text {. }
$$

This system can be referred to as the drive system or, as being called in the communication system, the transmitter.

Another chaotic system is described as

$$
\dot{Y}=F^{\prime}(Y, t)+G,
$$

where $G$ is the controller. Usually, this system is called the response system or, as being referred to in the communication system, the receiving system. Here $t$ is the time, and 


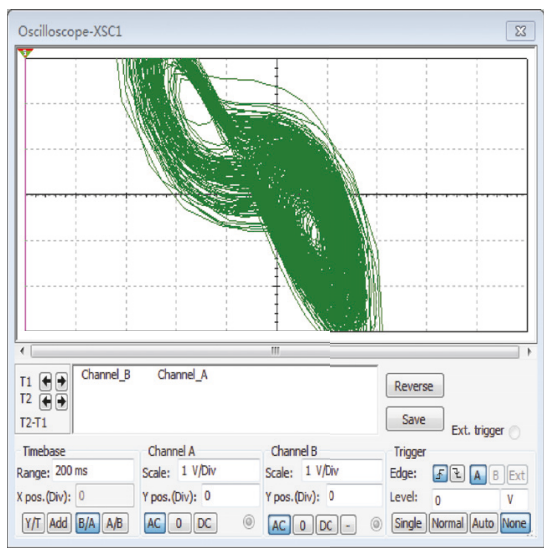

(a) $x y$ phase portrait

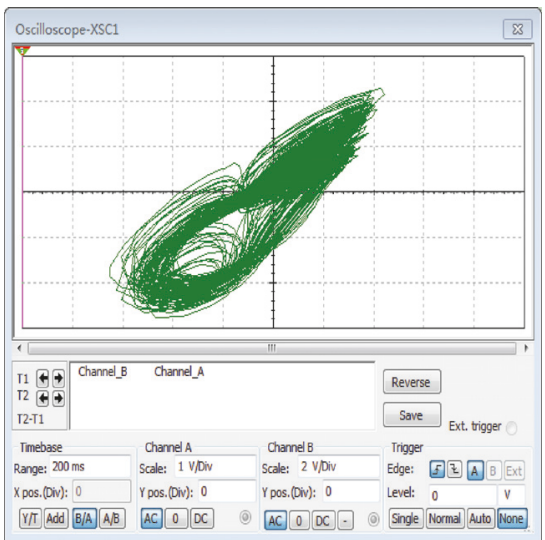

(d) $x u$ phase portrait

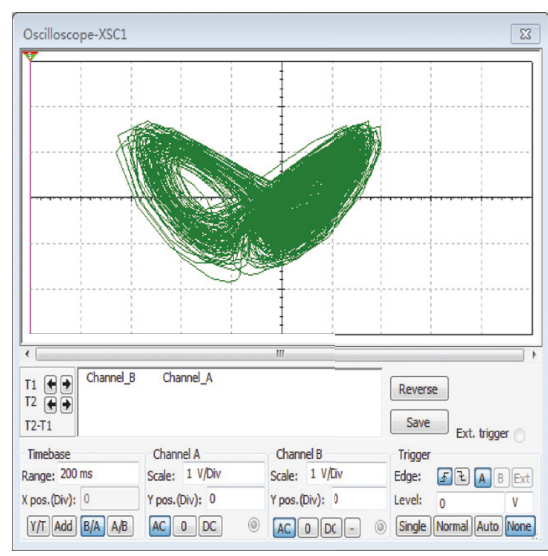

(b) $x z$ phase portrait

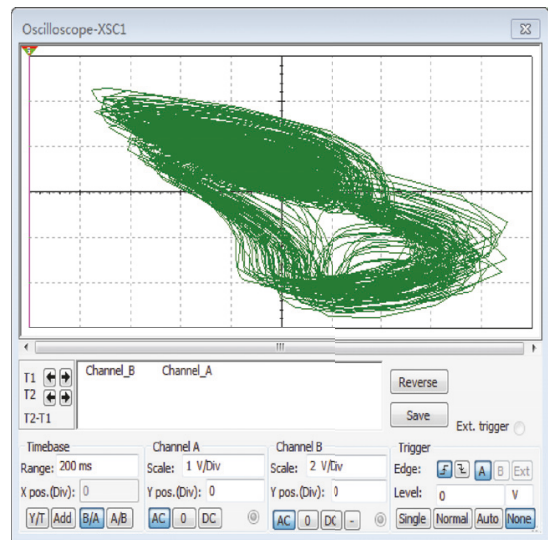

(e) $y u$ phase portrait

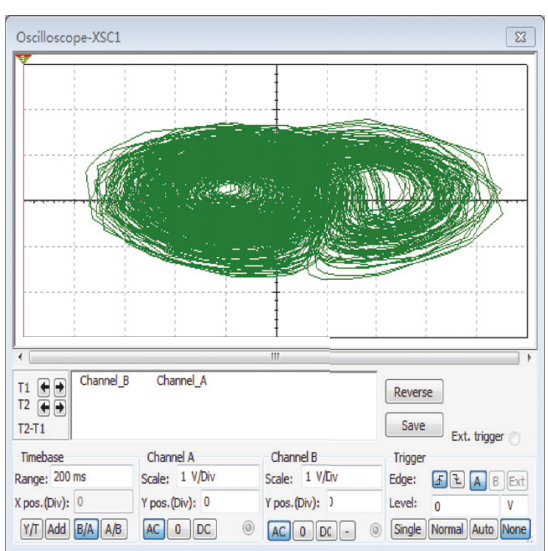

(c) $z y$ phase portrait

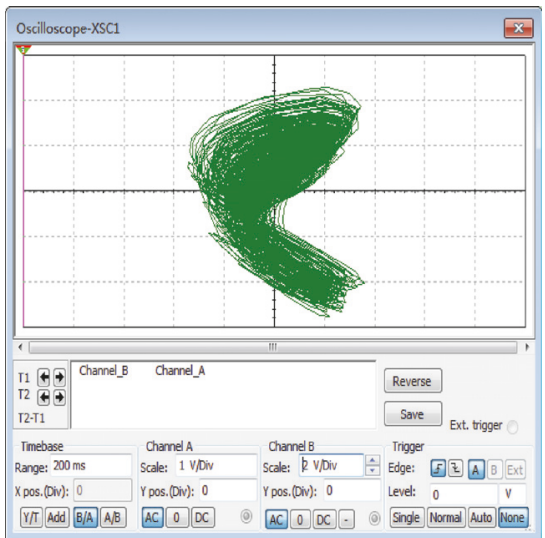

(f) $z u$ phase portrait

Figure 6: $(y+z)-u-x$ hyperchaotic phase portraits with Multisim.

vectors $X, Y \in R^{n}$. They have dimensional components $\left(x_{1}, x_{2}, \ldots, x_{n}\right)$ and $\left(y_{1}, y_{2}, \ldots, y_{n}\right)$, respectively. The two chaotic systems can be the same or different, but their initial conditions are different. If the two chaotic systems are connected in some way by the controller, we consider $X\left(t ; t_{0}, X_{0}\right)$ and $Y\left(t ; t_{0}, Y_{0}\right)$ are the solutions of system (15) and system (16), respectively, and satisfy the smooth condition of the function. If $R^{n}$ has a subset of $D\left(t_{0}\right)$ and the initial value $X_{0}, Y_{0} \in D\left(t_{0}\right)$, then, when $t \rightarrow \infty$, the following exists:

$$
\zeta \equiv \lim _{t \rightarrow \infty}\left\|X\left(t ; t_{0}, X_{0}\right)-Y\left(t ; t_{0}, Y_{0}\right)\right\| \longrightarrow 0
$$

Then, the response system (15) is synchronized with the drive system (16). In short, the synchronization error system of the chaotic systems is defined as $\dot{e}=\dot{y}-\dot{x}$. Chaotic synchronization means the asymptotic stability of the error system for the drive system and the response system at the origin; that is, $\lim _{t \rightarrow \infty}\|e(t)\| \rightarrow 0$. Obviously, the controller plays a key role, and the goal of the controller is to stabilize the synchronization error system at the origin. Therefore, through the design of a variety of different controllers, there will be a variety of synchronization methods.
Thus, we consider the novel drive system described by

$$
\begin{aligned}
& \dot{x}_{1}=-x_{1}-x_{2}-a x_{4} \\
& \dot{x}_{2}=-b x_{1}+c x_{1} x_{3} \\
& \dot{x}_{3}=-d x_{3}-e x_{1} x_{2} \\
& \dot{x}_{4}=-x_{2}-x_{3}-e x_{4},
\end{aligned}
$$

where $x_{1}, x_{2}, x_{3}$, and $x_{4}$ are the states and $a, b, c, d$, and $e$ are system parameters. When choosing $a=0.1, b=4.55, c=$ $1.96, d=0.37$, and $e=0.5$, the novel system (18) is chaotic. Next, we consider the novel response system described by

$$
\begin{aligned}
& \dot{y}_{1}=-y_{1}-y_{2}-a y_{4}+u_{1} \\
& \dot{y}_{2}=-b y_{1}+c y_{1} y_{3}+u_{2} \\
& \dot{y}_{3}=-d y_{3}-e y_{1} y_{2}+u_{3} \\
& \dot{y}_{4}=-y_{2}-y_{3}-e y_{4}+u_{4},
\end{aligned}
$$

where $y_{1}, y_{2}, y_{3}, y_{4}$ are the states and $u_{1}, u_{2}, u_{3}, u_{4}$ are the controllers to be designed so as to achieve global chaos synchronization between systems (18) and (19). 


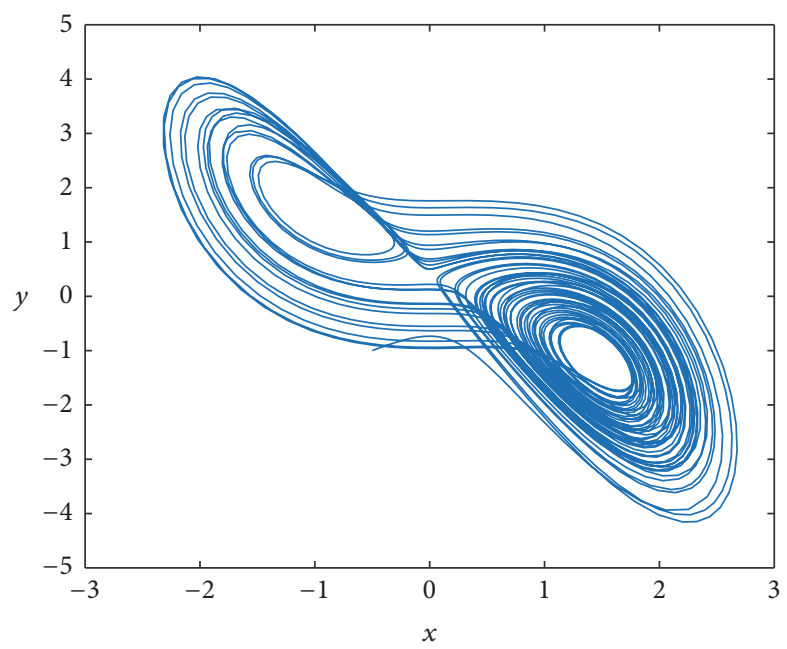

(a) $x y$ plane

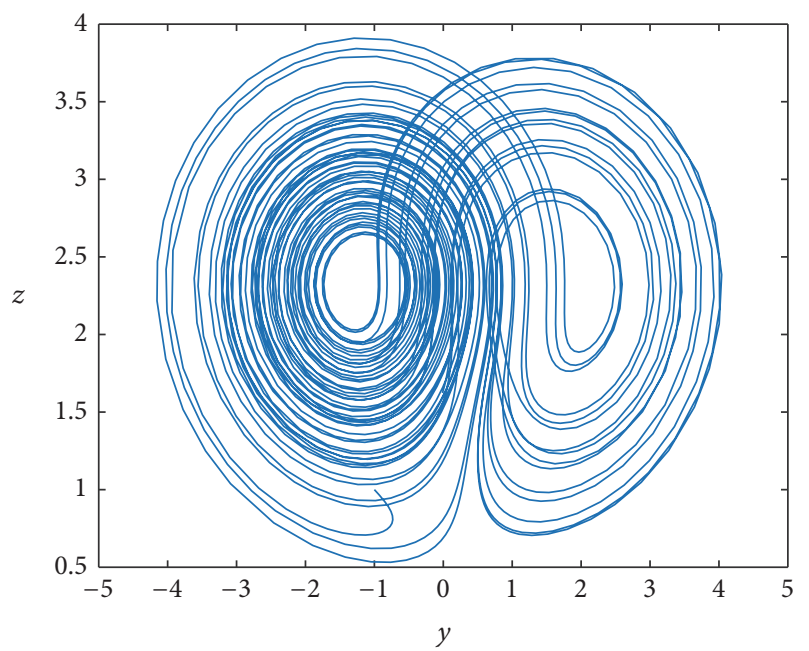

(c) $z y$ plane

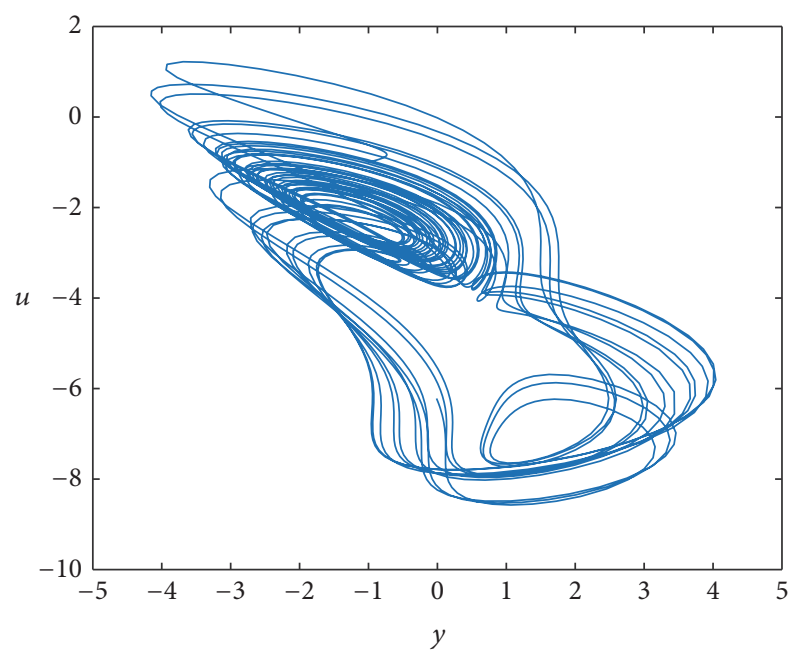

(e) $y u$ plane

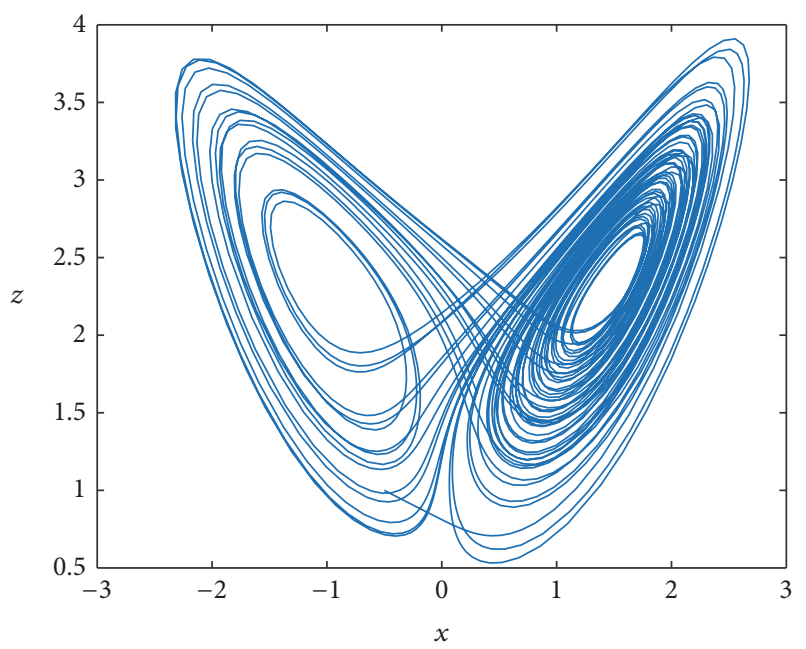

(b) $x z$ plane

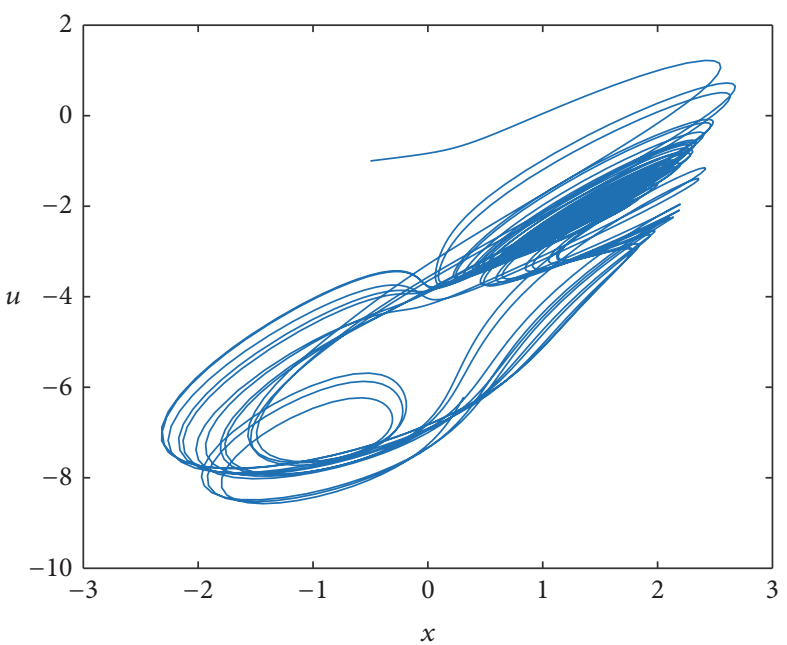

(d) $x u$ plane

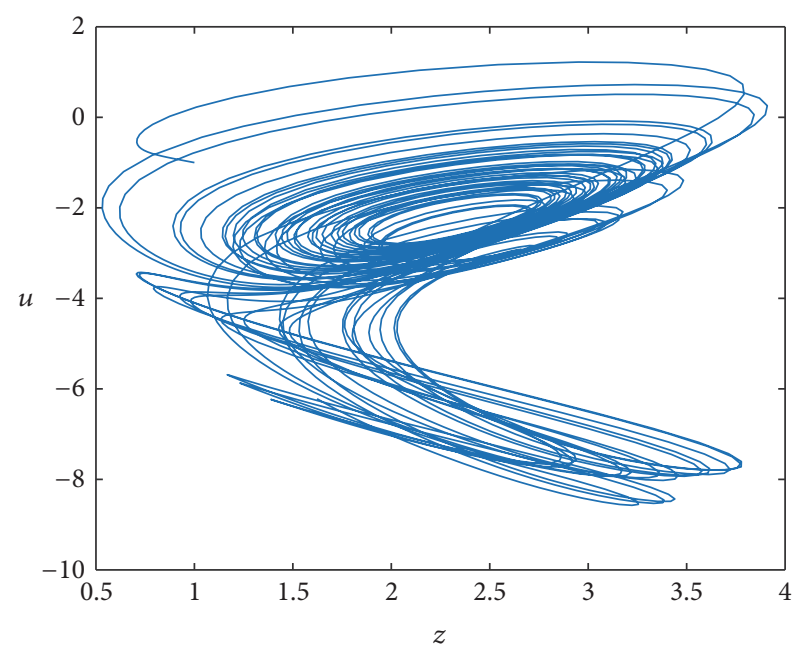

(f) $z u$ plane

FIgURE 7: $(y+z)-u-x$ hyperchaotic phase portraits with Matlab. 


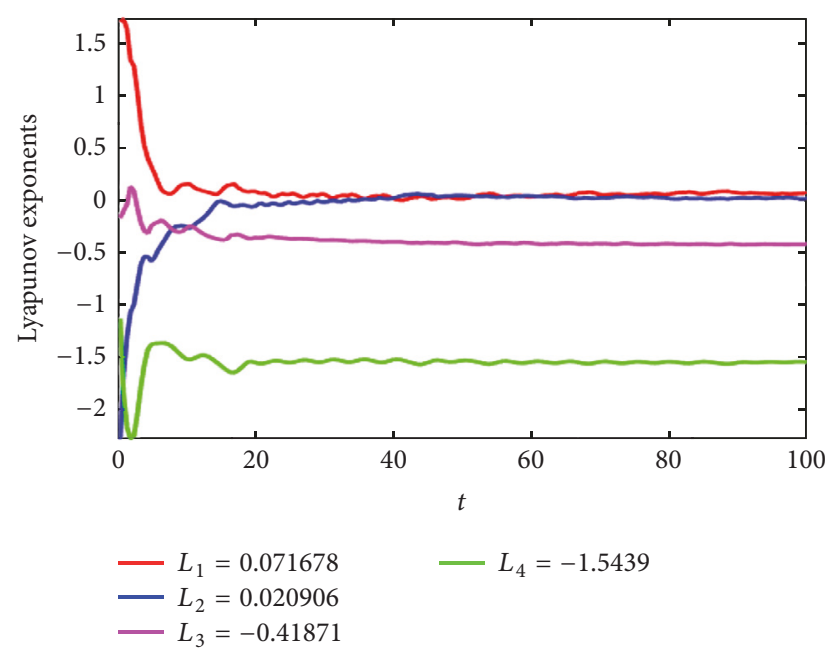

FIGURE 8: Lyapunov exponents of the new system (6).

The synchronization error is defined as

$$
\dot{\xi}_{i}=\dot{y}_{i}-\dot{x}_{i}, \quad(i=1,2,3,4) .
$$

Then, the synchronization error system between the drive system (18) and the response system (19) is easily obtained as

$$
\begin{aligned}
& \dot{\xi}_{1}=-\xi_{1}-\xi_{2}-a \xi_{4}+u_{1} \\
& \dot{\xi}_{2}=-b \xi_{1}+c\left(y_{1} y_{3}-x_{1} x_{3}\right)+u_{2} \\
& \dot{\xi}_{3}=-d \xi_{3}-e\left(y_{1} y_{2}-x_{1} x_{2}\right)+u_{3} \\
& \dot{\xi}_{4}=-\xi_{2}-\xi_{3}-e \xi_{4}+u_{4} .
\end{aligned}
$$

Construct the following controller system:

$$
\begin{aligned}
& u_{1}=\xi_{1}+\xi_{2}+a \xi_{4}-k_{1} \xi_{1} \\
& u_{2}=b \xi_{1}-c\left(y_{1} y_{3}-x_{1} x_{3}\right)-k_{2} \xi_{2} \\
& u_{3}=d \xi_{3}+e\left(y_{1} y_{2}-x_{1} x_{2}\right)-k_{3} \xi_{3} \\
& u_{4}=\xi_{2}+\xi_{3}+e \xi_{4}-k_{4} \xi_{4},
\end{aligned}
$$

where $k_{i}>0(i=1,2,3,4)$, for the use of controlling the synchronization speed. Substituting (22) into (21), we can obtain the error dynamics:

$$
\begin{aligned}
& \dot{\xi}_{1}=-k_{1} \xi_{1} \\
& \dot{\xi}_{2}=-k_{2} \xi_{2} \\
& \dot{\xi}_{3}=-k_{3} \xi_{3} \\
& \dot{\xi}_{4}=-k_{4} \xi_{4} .
\end{aligned}
$$

Then, the Lyapunov function $V$ is considered as

$$
V=\frac{\left(\xi_{1}^{2}+\xi_{2}^{2}+\xi_{3}^{2}+\xi_{4}^{2}\right)}{2} .
$$

Clearly, $V$ is a positive definite function. Differentiating $V$ from (24), we can obtain

$$
\begin{aligned}
\dot{V}= & \xi_{1} \dot{\xi}_{1}+\xi_{2} \dot{\xi}_{2}+\xi_{3} \dot{\xi}_{3}+\xi_{4} \dot{\xi}_{4} \\
= & \xi_{1}\left(-k_{1} \xi_{1}\right)+\xi_{2}\left(-k_{2} \xi_{2}\right)+\xi_{3}\left(-k_{3} \xi_{3}\right) \\
& +\xi_{4}\left(-k_{4} \xi_{4}\right)=-k_{1} \xi_{1}^{2}-k_{2} \xi_{2}^{2}-k_{3} \xi_{3}^{2}-k_{4} \xi_{4}^{2} .
\end{aligned}
$$

Thus, we can easily obtain $\dot{V}=-k_{1} \xi_{1}^{2}-k_{2} \xi_{2}^{2}-k_{3} \xi_{3}^{2}-k_{4} \xi_{4}^{2} \leq$ 0 , which shows that $\dot{V}$ is a negative semidefinite function. According to the Lyapunov stability theory, it follows that if $V$ is a positive definite function and $\dot{V}$ is a negative semidefinite function, then the system is consistent and stable at the origin of the equilibrium state. Hence, it follows that $\xi_{1}(t) \rightarrow 0$, $\xi_{2}(t) \rightarrow 0, \xi_{3}(t) \rightarrow 0$, and $\xi_{4}(t) \rightarrow 0$ exponentially as $t \rightarrow \infty$. That is, the active synchronization error system (21) is asymptotically stable at the origin and the synchronization is effectively realized. This completes the proof.

For the numerical simulations, the fourth-order RungeKutta method is used to solve the novel drive system (18) and the novel response system (19) with active control method. In the following simulations, suppose that the initial values of the novel drive system are chosen as $x_{1}(0)=-14, x_{2}(0)=-5$, $x_{3}(0)=43$, and $x_{4}(0)=0.3$. The initial values of the novel response system are chosen as $y_{1}(0)=-12, y_{2}(0)=-3$, $y_{3}(0)=35$, and $y_{4}(0)=0.2$. The control gains are chosen as $k_{1}=1, k_{2}=0.5, k_{3}=0.8$, and $k_{4}=0.5$. The history of the synchronization errors is shown in Figure 9. It can be seen from Figure 9 that the synchronization errors $\xi_{1}, \xi_{2}$, $\xi_{3}, \xi_{4}$ are asymptotically stabilized at the origin. The timing diagrams of $x_{1}-y_{1}, x_{2}-y_{2}, x_{3}-y_{3}, x_{4}-y_{4}$ are shown in Figure 10. The synchronous waveforms of the two chaotic systems are shown to be the same in a very short period of time; thus the active control synchronization is implemented.

The advantages of active control method are presented as follows: this method is a simple but applicable tool for analyzing synchronization stability, and the synchronization speed is very fast. In practical applications, it has always been known that the less the control signal is, the more easily the hardware circuit of the control process is realized. Therefore, the active control synchronization method is easier to be realized in the hardware circuits because of its less control signal and lower cost compared with other control methods [20-22]. By the use of this control method in chaotic secure communication, the number of signals transmitted through the public channel can be greatly decreased to further guarantee the security and good robustness. Thus, it is easy to be implemented in engineering. But the disadvantage of this control method is that the anti-interference ability is weaker.

\section{Applications of the Novel Hyperchaotic System}

4.1. Circuit Deformation. In the following, another new fourth-order hyperchaotic circuit is constructed successfully through circuit deformation based on the novel $(y+z)-u-x$ 


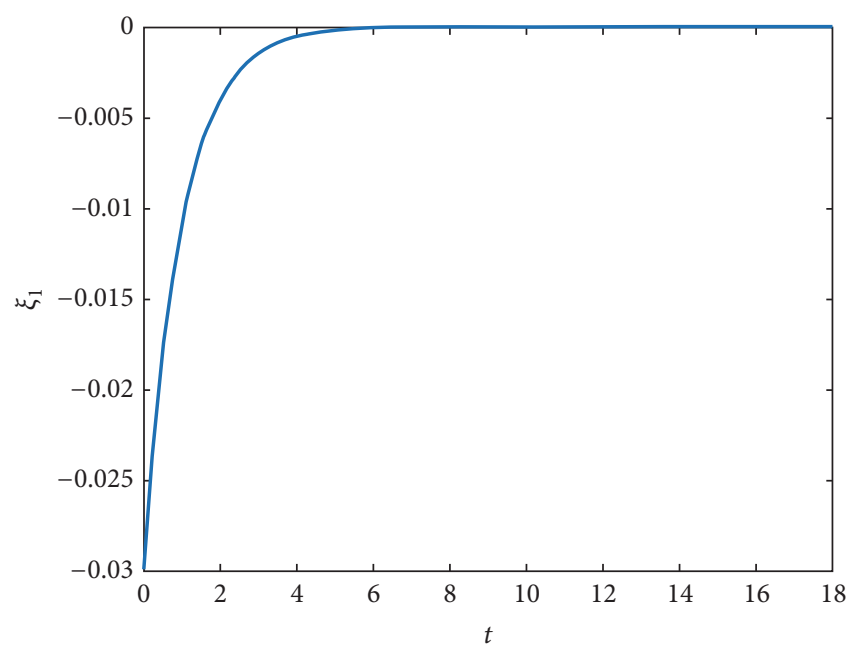

(a) Synchronization error $\xi_{1}$

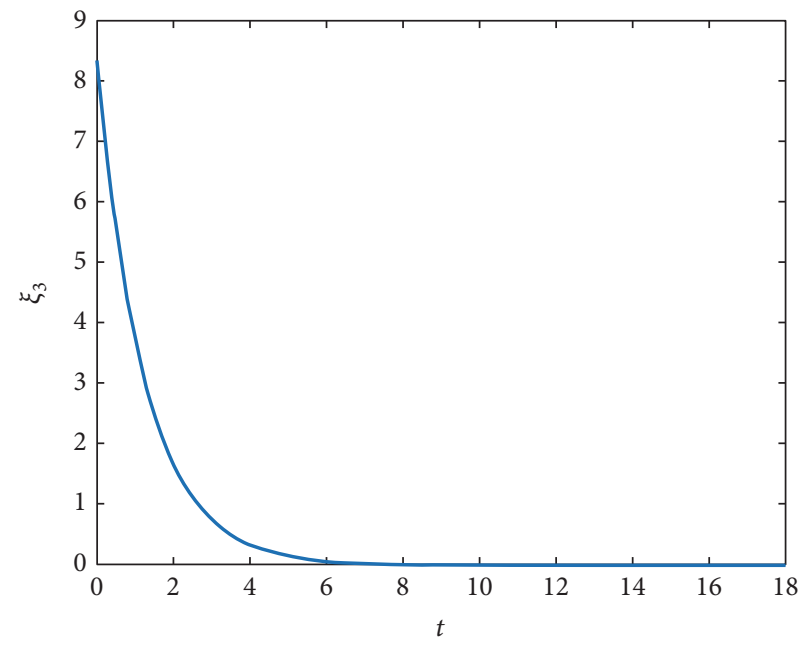

(c) Synchronization error $\xi_{3}$

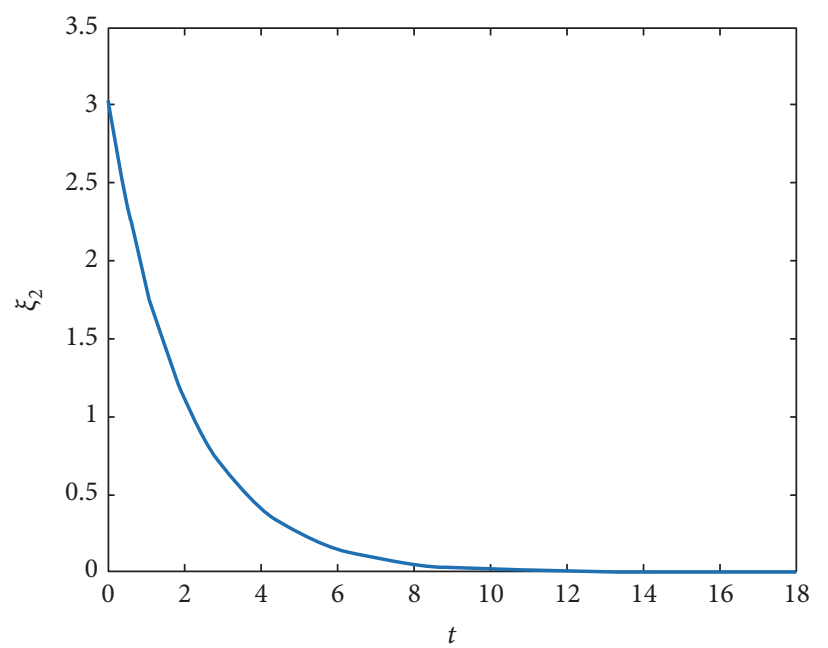

(b) Synchronization error $\xi_{2}$

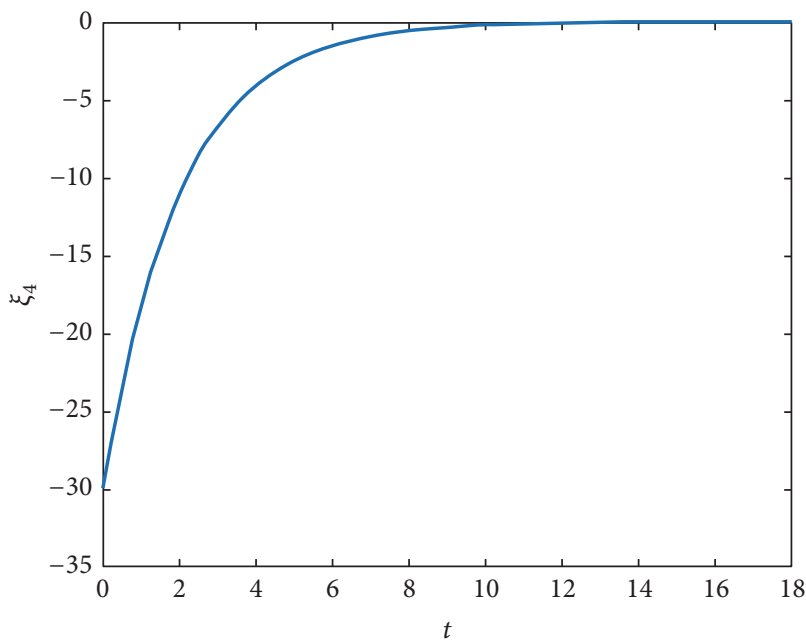

(d) Synchronization error $\xi_{4}$

FIGURE 9: The history of synchronization errors.

hyperchaotic circuit. The circuit deformation principle is given as follows: according to the fourth-order $(y+z)-u-x$ hyperchaotic circuit, the input is introduced from the $z$ end, and feedback is given to the $y$ input stage after the $u$ stage. Thus, another novel fourth-order hyperchaotic circuit is proposed, and the novel hyperchaotic circuit schematic is shown in Figure 11. It is composed of 5 operational amplifiers, 2 analog multipliers, 11 resistors, and 4 capacitors. In order to facilitate the narrative, we call this novel fourth-order circuit $z-u-y$ hyperchaotic circuit.

The beneficial effects of the novel fourth-order $z-u-y$ hyperchaotic circuit are as follows: it not only can output four chaotic waveforms and six phase portraits, but also can output upright phase portrait shape like butterfly wings and stable fourth-order double vortex chaotic signals. Also, operational amplifier TL082 or TL084 and analog multiplier AD633 are used. According to the novel $z-u-y$ hyperchaotic circuit shown in Figure 11, the normalized resistor is set as
$R=100 \mathrm{k} \Omega$. Thus, the state equation of the corresponding $z-u-y$ hyperchaotic circuit is obtained as follows:

$$
\begin{aligned}
& \dot{x}=-\frac{100 \mathrm{k}}{100 \mathrm{k}} x-\frac{100 \mathrm{k}}{100 \mathrm{k}} y \\
& \dot{y}=-\frac{100 \mathrm{k}}{22 \mathrm{k}} x+\frac{100 \mathrm{k}}{510 \Omega} \times 0.01 x z-\frac{100 \mathrm{k}}{100 \mathrm{k}} \times 0.1 u \\
& \dot{z}=-\frac{100 \mathrm{k}}{270 \mathrm{k}} z-\frac{100 \mathrm{k}}{2 \mathrm{k}} \times 0.01 x y \\
& \dot{u}=-\frac{100 \mathrm{k}}{100 \mathrm{k}} y-\frac{100 \mathrm{k}}{20 \mathrm{k}} \times 0.1 u .
\end{aligned}
$$

Then, another novel fourth-order hyperchaotic system is obtained by (26):

$$
\begin{aligned}
& \dot{x}=-x-y \\
& \dot{y}=-a x+b x z-c u
\end{aligned}
$$




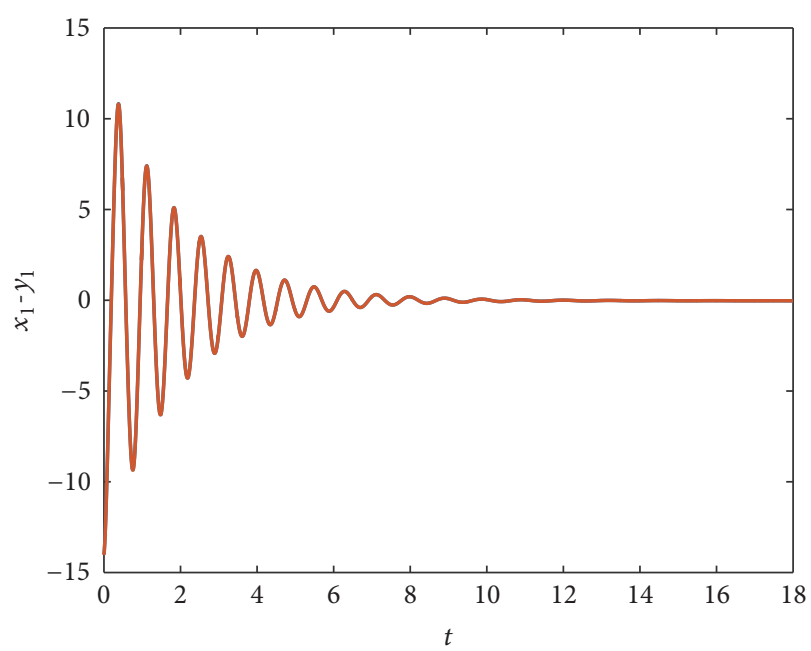

$x_{1}$
$-y_{1}$

(a) Synchronization of $x_{1}-y_{1}$

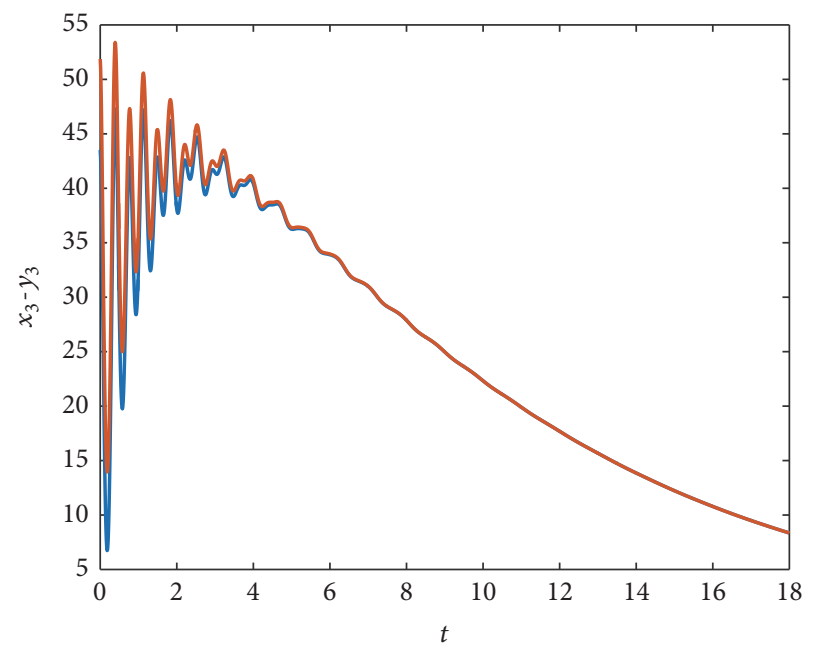

$x_{3}$
$-y_{3}$

(c) Synchronization of $x_{3}-y_{3}$

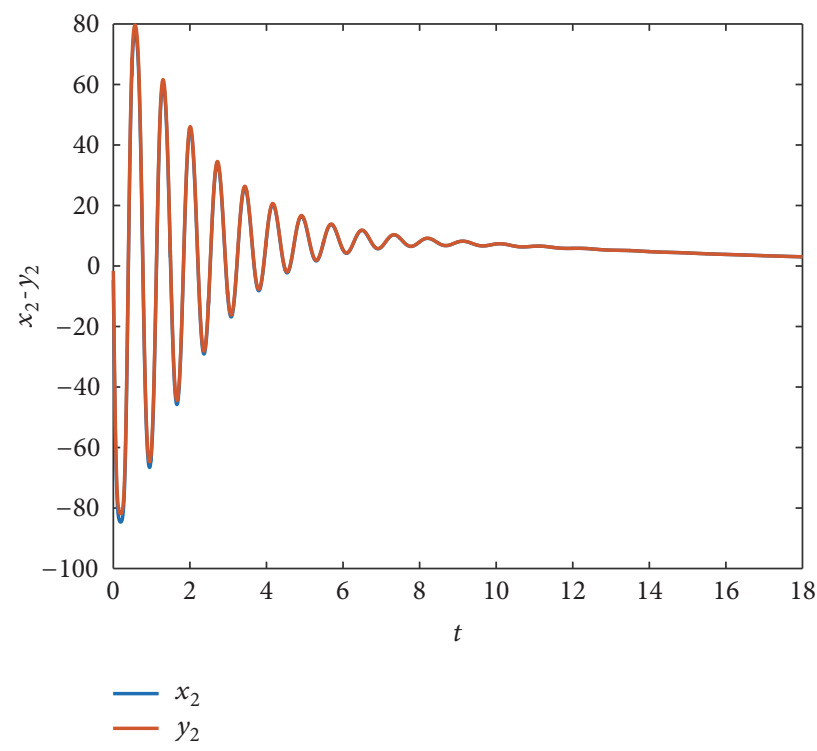

(b) Synchronization of $x_{2}-y_{2}$

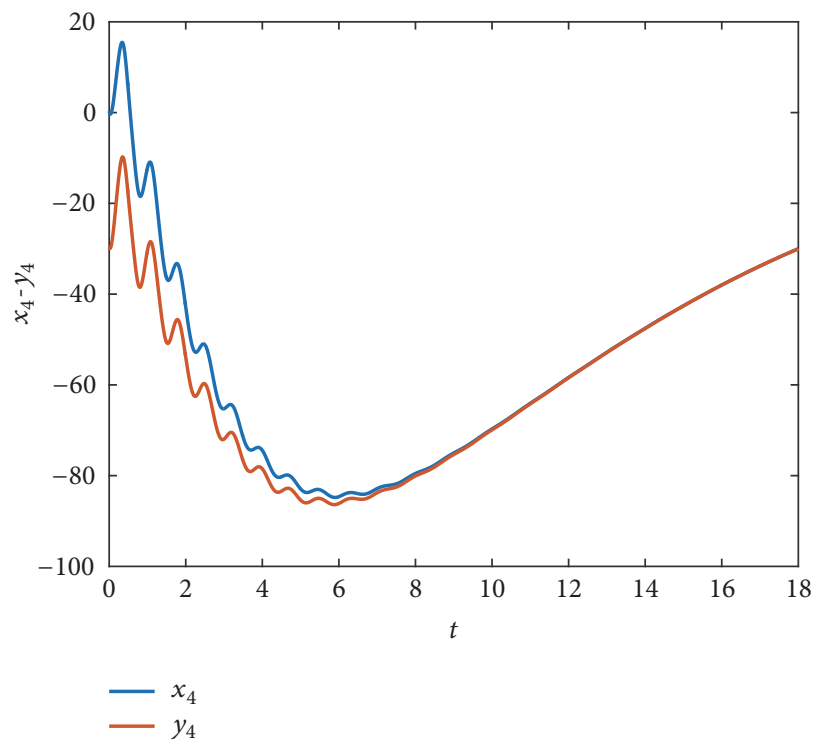

(d) Synchronization of $x_{4}-y_{4}$

FIGURE 10: Synchronization of the two chaotic systems.

$$
\begin{aligned}
& \dot{z}=-d z-e x y \\
& \dot{u}=-y-e u .
\end{aligned}
$$

When choosing $a=4.55, b=1.96, c=0.1, d=0.37$, and $e=$ 0.5 , the novel system (27) is chaotic. Substituting the specific parameter values, (27) becomes

$$
\begin{aligned}
& \dot{x}=-x-y \\
& \dot{y}=-4.55 x+1.96 x z-0.1 u \\
& \dot{z}=-0.37 z-0.5 x y \\
& \dot{u}=-y-0.5 u .
\end{aligned}
$$

This is another novel hyperchaotic system through circuit deformation based on the $(y+z)-u-x$ hyperchaotic circuit. Similarly, the divergence of the novel chaotic system (27) is easily calculated as

$$
\nabla=\frac{\partial \dot{x}}{\partial x}+\frac{\partial \dot{y}}{\partial y}+\frac{\partial \dot{z}}{\partial z}+\frac{\partial \dot{u}}{\partial u}=-1-d-e<0 .
$$

The divergence is less than 0 . A necessary and sufficient condition for system (27) to be dissipative is that the divergence of the vector field is negative when the time tends to be infinite, and the corresponding dynamic characteristics will be presented.

All of the electronic components are easily available. In order to illustrate the effectiveness of the proposed $z-u-y$ 


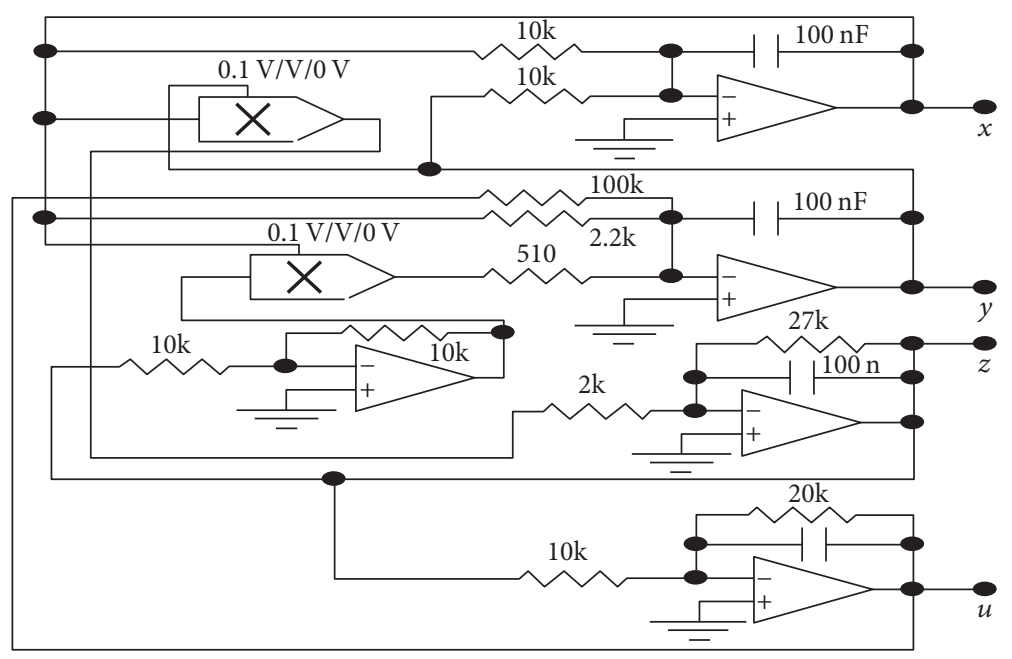

FIGURE 11: $z-u-y$ hyperchaotic circuit schematic.

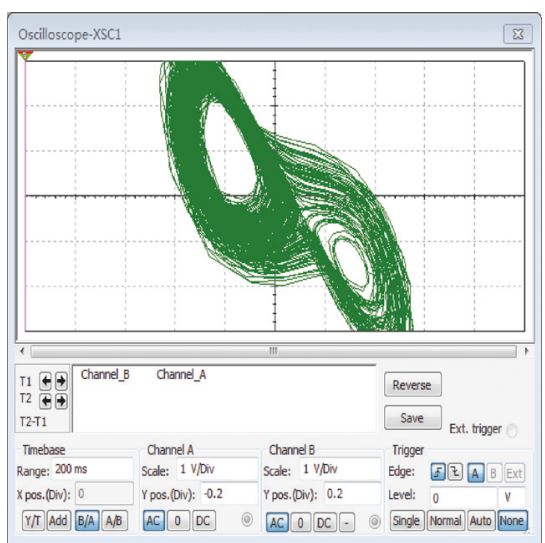

(a) $x y$ phase portrait

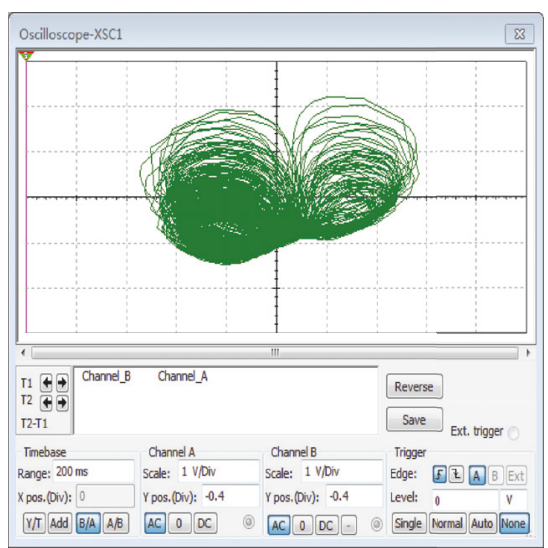

(d) $x u$ phase portrait

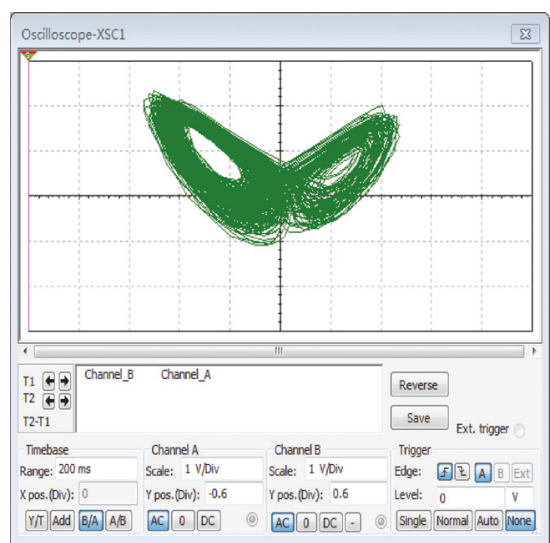

(b) $x z$ phase portrait

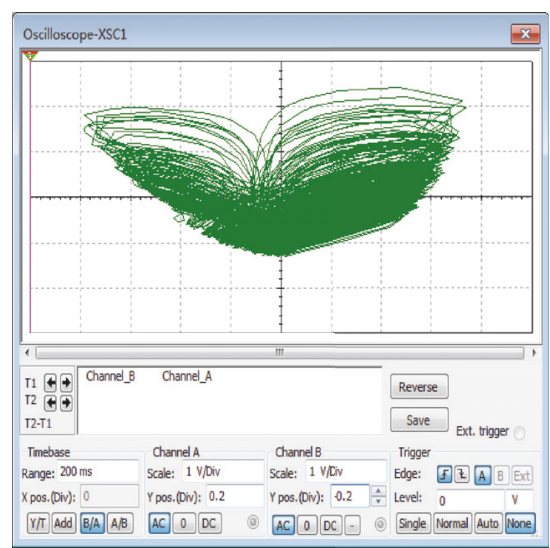

(e) $y u$ phase portrait

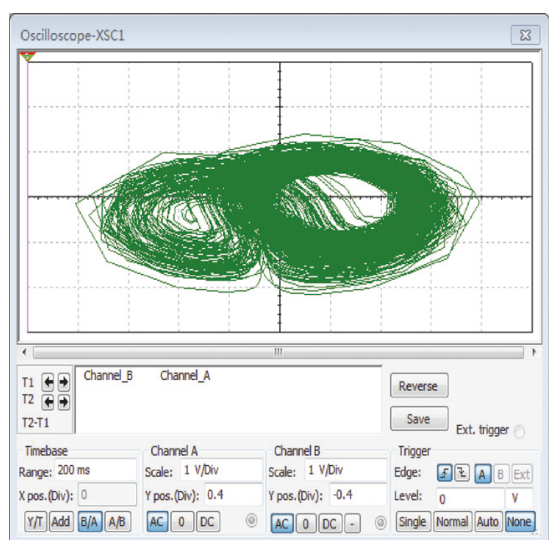

(c) $z y$ phase portrait

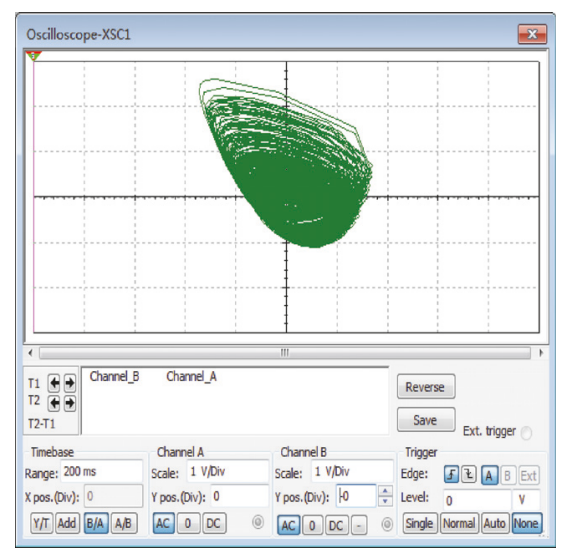

(f) $z u$ phase portrait

FIGURE 12: $z-u-y$ hyperchaotic phase portraits with Multisim.

hyperchaotic circuit, some simulations are presented by Multisim. The $z-u-y$ hyperchaotic phase portraits are shown in Figure 12 with Multisim. It can be seen from the simulation results that it can output six chaotic phase portraits of $x y$, $x z, z y, x u, y u$, and $z u$. A variety of chaotic signals can be displayed on the oscilloscope. Similarly, other kinds of experiments can also be implemented through the novel $z-u$ - $y$ hyperchaotic circuit. 


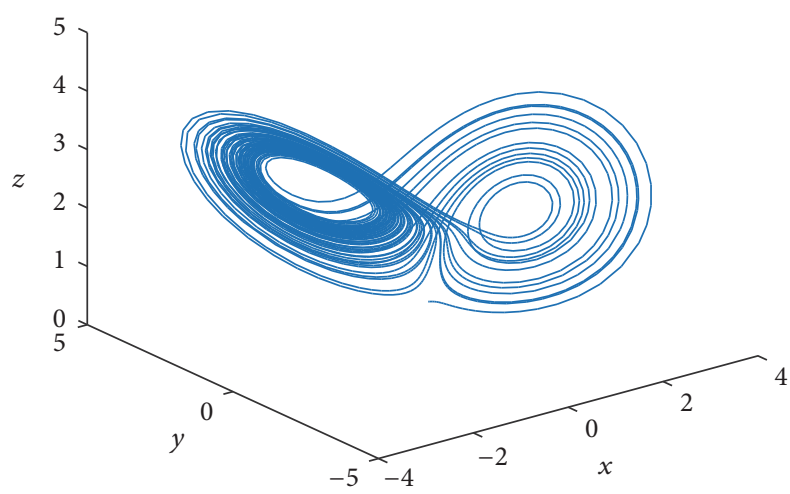

FIGURE 13: Three-dimensional chaotic attractor.

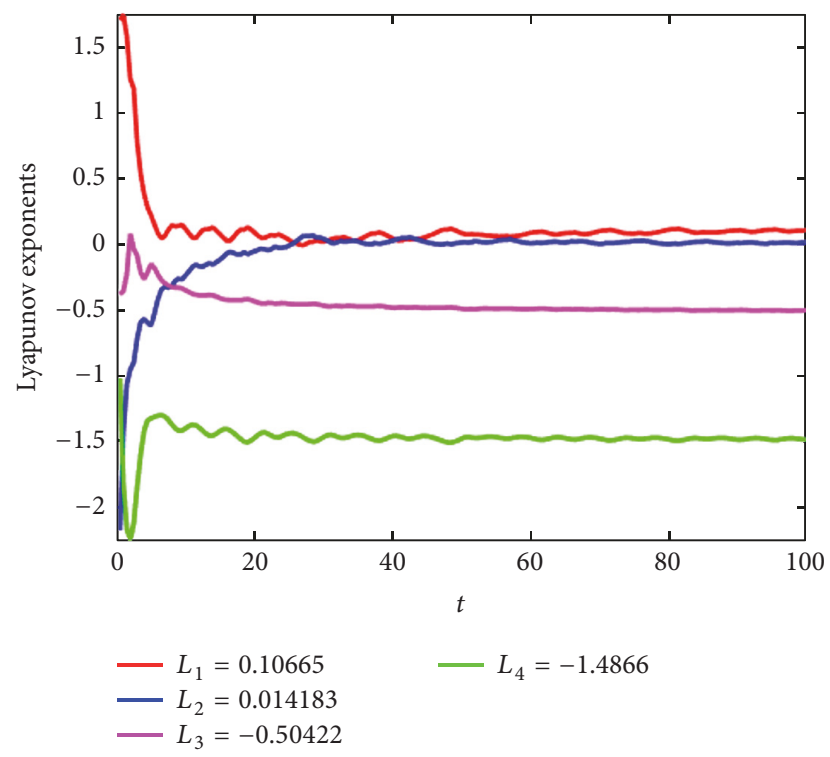

FIGURE 14: Lyapunov exponents of system (28).

In order to further study the chaotic dynamical behavior of the novel $z-u-y$ hyperchaotic system (27), some numerical analysis and simulations are implemented. The threedimensional chaotic attractor of the $z-u-y$ hyperchaotic system is shown in Figure 13. And the Lyapunov exponents of the novel $z-u-y$ hyperchaotic system are also strictly calculated. With the parameters chosen as $a=4.55, b=1.96$, $c=0.1, d=0.37$, and $e=0.5$, the corresponding Lyapunov exponents are obtained as $L_{1}=0.1067, L_{2}=0.0142, L_{3}=$ -0.5042 , and $L_{4}=-1.4866$.

This means that system (28) is a hyperchaotic system since $L_{1}$ and $L_{2}$ are positive values and a dissipative system since the sum of the Lyapunov exponents is negative. It meets the condition of at least two positive Lyapunov exponents for the hyperchaotic systems. And the corresponding Lyapunov exponents diagram of the novel hyperchaotic system (28) is shown in Figure 14. It is further verified that the novel system (28) is a hyperchaotic system.

4.2. Hyperchaotic Secure Communication. It is well known that the hyperchaotic signals are especially suitable for the secure communication field. In order to improve the security of secure communication system, it is considered that the novel hyperchaotic system should be selected as the chaotic system. In the proposed hyperchaotic secure communication scheme, the hyperchaotic secure communication circuit is implemented by using some electronic components containing analog multipliers, operational amplifiers, resistors, and capacitors with a novel hyperchaotic system as chaos generator. On the basis of the proposed fourth-order $(y+z)-u-x$ hyperchaotic circuit, the hyperchaotic secure communication circuit schematic by Multisim is shown in Figure 15. Its circuit principle is carefully presented as follows.

It consists of 12 operational amplifiers together with 4 analog multipliers. Its basic circuit is composed of two identical $(y+z)-u-x$ hyperchaotic circuit units with a little change. The left side of the circuit is the transmitting system and the right side is the receiving system. The inverting input end of transmitter-modulator is connected with the transmitted signal to be transmitted. The same-phase input end is connected with the $x$ output terminal of the novel $(y+z)-u-x$ hyperchaotic circuit. The inverting input end of receiver-demodulator is connected with the communication channel signal, and the same-phase input end is connected with $x$ output end of the novel $(y+z)-u-x$ hyperchaotic circuit. The signal at the output is the output of the receiver. In this way the receiving system is more easily synchronized with the transmitting system and the robustness of the $(y+z)-u-x$ hyperchaotic circuit is maintained. This method can effectively prevent the useful information from being intercepted in the communication process [34].

The advantages of the novel hyperchaotic secure communication circuit are described as follows: the implementation cost of the novel hyperchaotic system is reduced to improve the practicability of the secure communication system, the number of the state variables and transmission channels needed to access the drive system is also reduced, and the circuit structure of the designed control system is greatly simplified. In the following, some simulation experiments are presented to verify whether two identical parameters of the $(y+z)-u-x$ hyperchaotic circuits can effectively achieve the signal transmission and reception without distortion. For example, an input sine wave with amplitude of $1 \mathrm{~V}$ and frequency of $1 \mathrm{kHz}$ is given; the transmitting and receiving signal waveforms by Multisim are shown in Figure 16. It can be seen from the simulation results that, no matter what kinds of signals are input, the full synchronization can be realized in two identical $(y+z)-u-x$ chaotic circuits if the component parameters of the transmitting circuit are completely consistent with the receiving circuit. Negligible distortion can be observed.

To illustrate the effectiveness of the proposed scheme, the intensity limit and stability of the transmitted signal, the broadband characteristics, and the accuracy requirements of electronic components are simulated by Multisim [34].

First of all, in order to verify whether the proposed hyperchaotic secure communication circuit has a choice for various input signals intensity, sine waves with frequency of $1 \mathrm{kHz}$ and amplitude of $100 \mathrm{mV}, 5 \mathrm{~V}, 15 \mathrm{~V}, 16 \mathrm{~V}$, and $25 \mathrm{~V}$ are input. It can be concluded from these waveforms that 


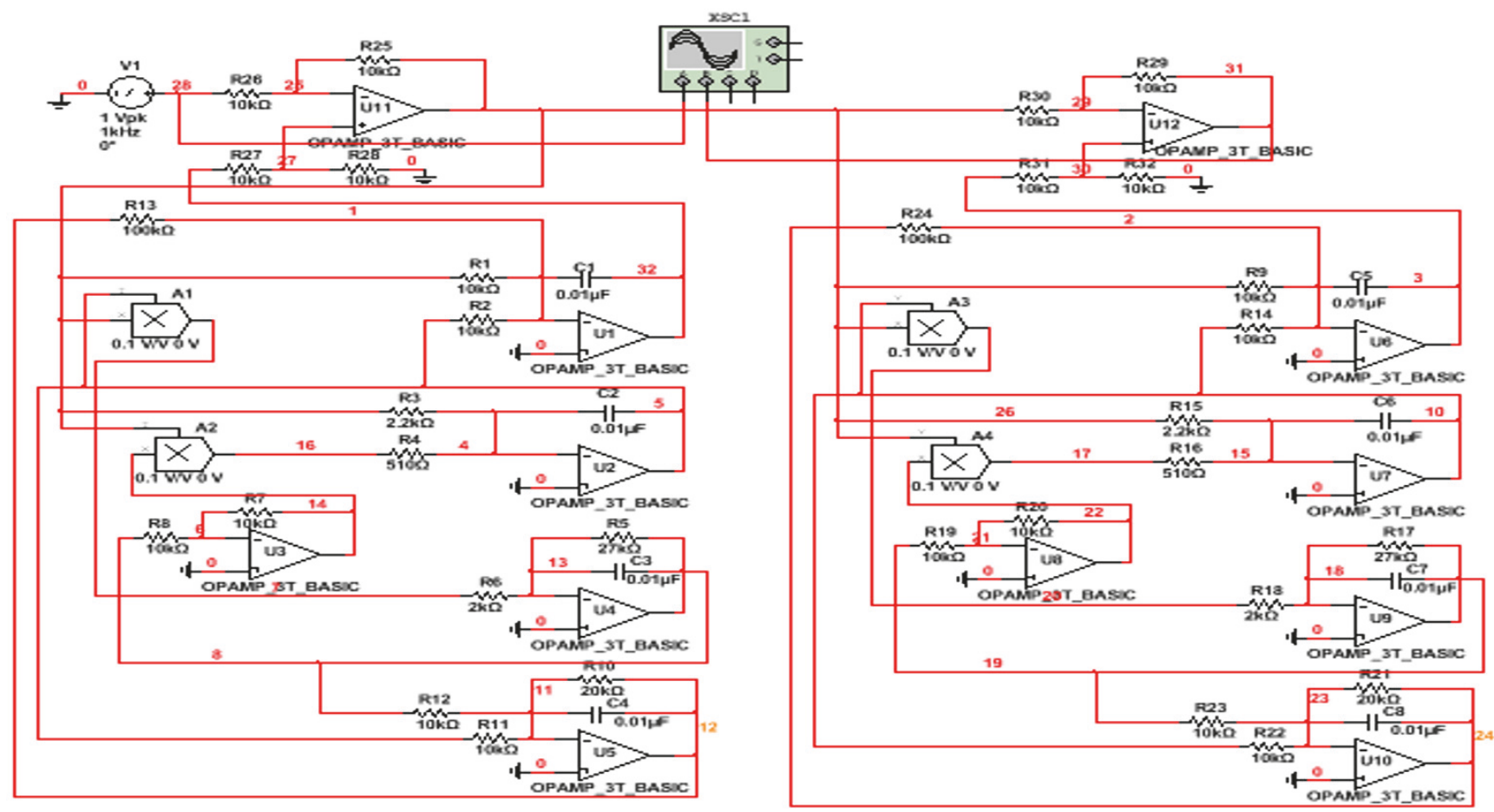

FIgURE 15: A novel hyperchaotic secure communication circuit by Multisim.

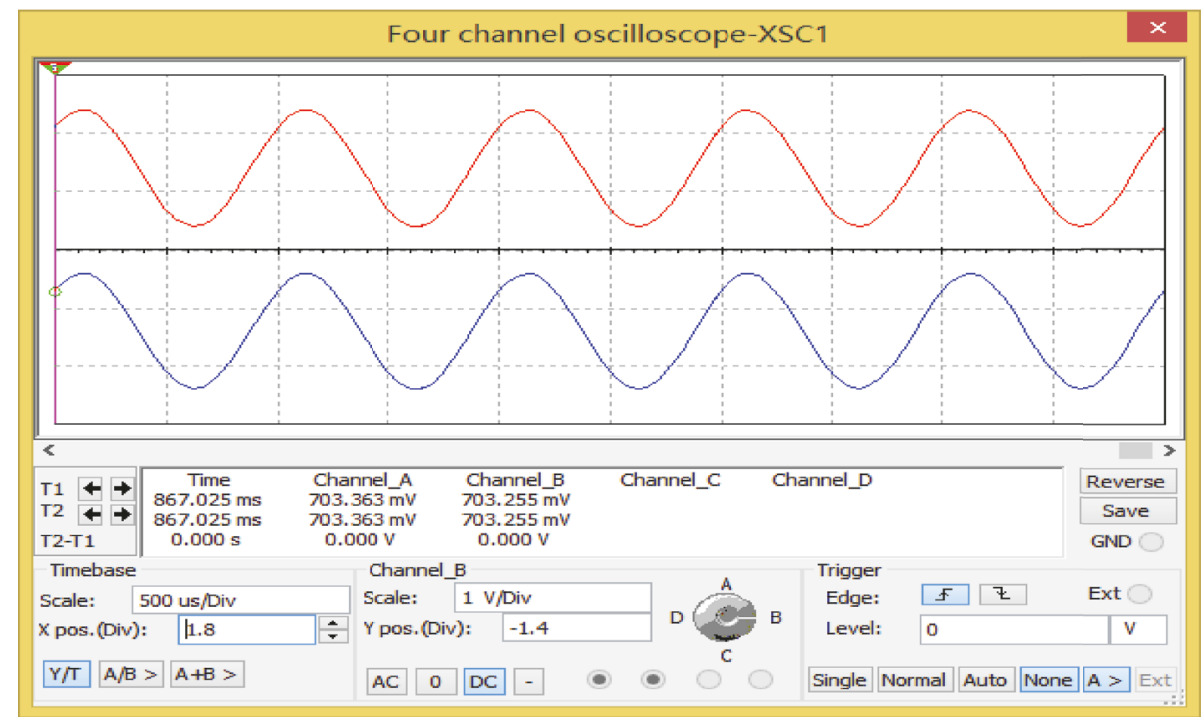

FIGURE 16: Transmitting and receiving waveforms with amplitude of $1 \mathrm{~V}$ and frequency of $1 \mathrm{kHz}$.

the signal transmission distortion will occur when the signal amplitude reaches $16 \mathrm{~V}$, as shown in Figures 17, 18, 19, and 20. When the signal amplitude exceeds $16 \mathrm{~V}$, the signal distortion is quite evident, as shown in Figures 21 and 22.

Secondly, in order to verify whether the proposed hyperchaotic secure communication circuit has a choice for the input signal frequency, sine waves with amplitude of $1 \mathrm{~V}$ and frequency of $100 \mathrm{~Hz}, 10 \mathrm{kHz}, 100 \mathrm{kHz}, 500 \mathrm{kHz}$, and $1 \mathrm{MHz}$ are given. It can be seen from these waveforms that it can transmit the signal from $1 \mathrm{~Hz}$ to $500 \mathrm{kHz}$ without distortion. This is due to the frequency limitations of the amplifiers, as already shown in [32]. When the signal frequency reaches $500 \mathrm{kHz}$ to $1 \mathrm{MHz}$, the signal distortion is quite evident, as shown in Figures 23, 24, 25, and 26. Thus, it can be concluded that the circuit has the characteristic of broadband.

From the simulation results above, we can draw the conclusion that the proposed hyperchaotic secure communication circuit based on the $(y+z)-u-x$ hyperchaotic 


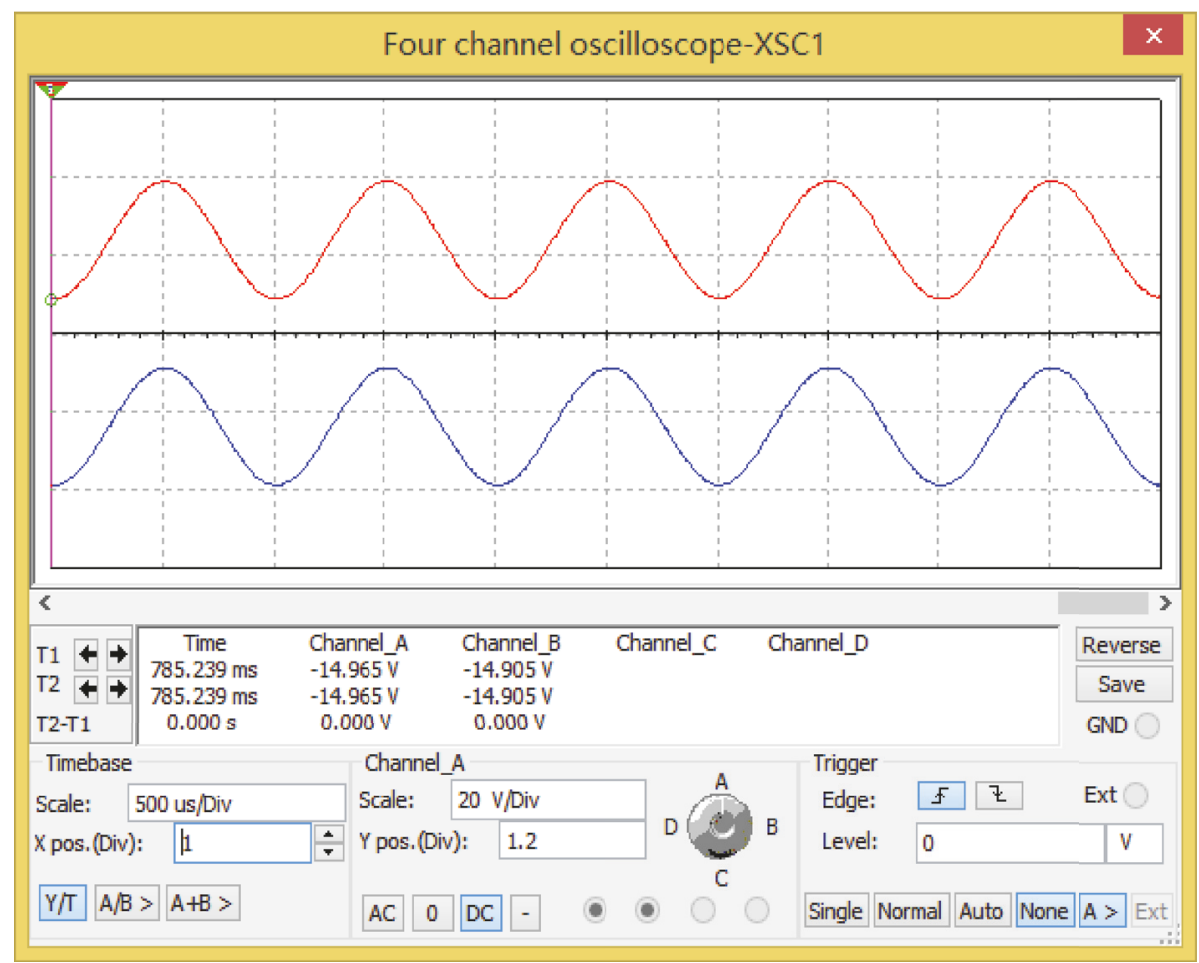

FIGURE 17: $15 \mathrm{~V}$.

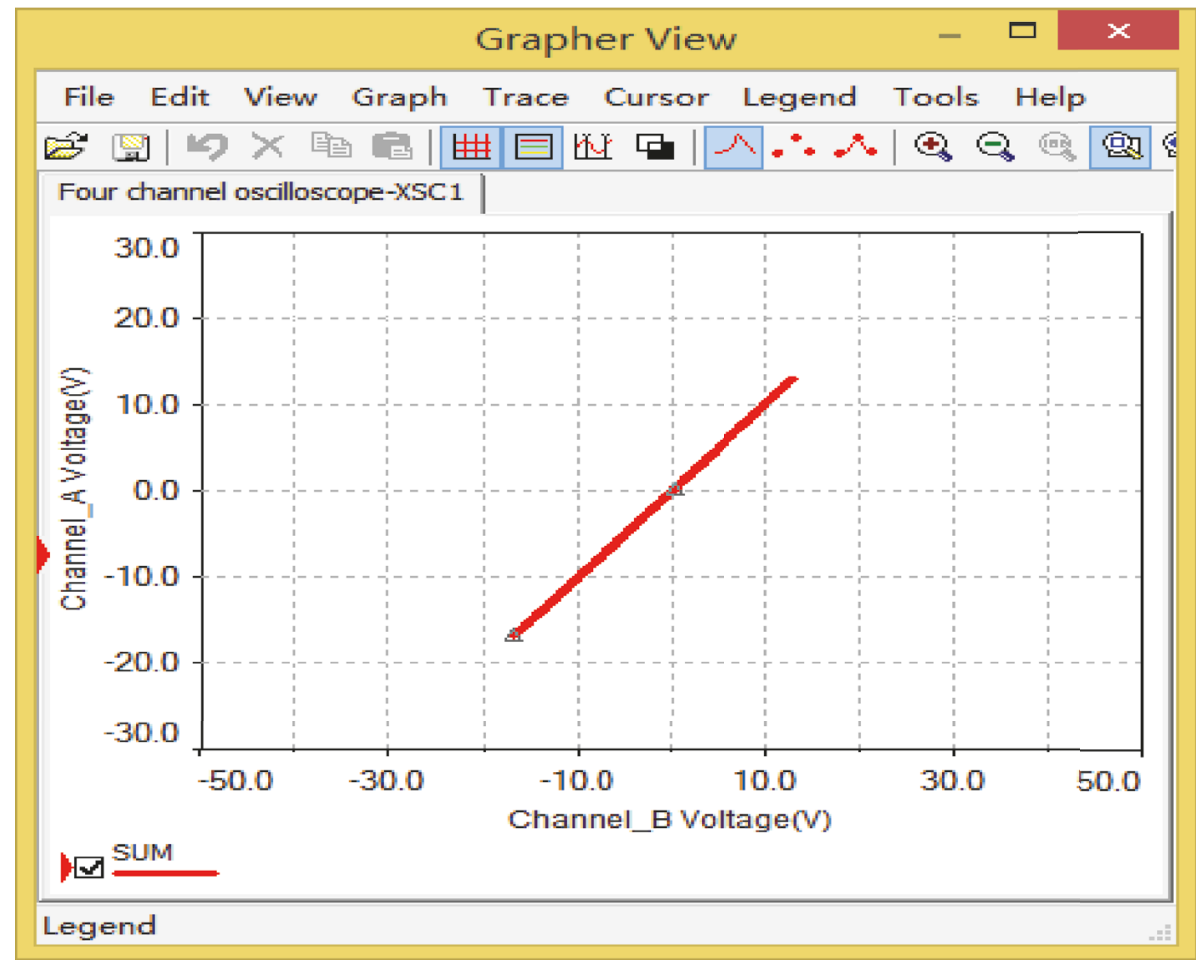

FIGURE 18: Synchronous phase portrait of $15 \mathrm{~V}$. 


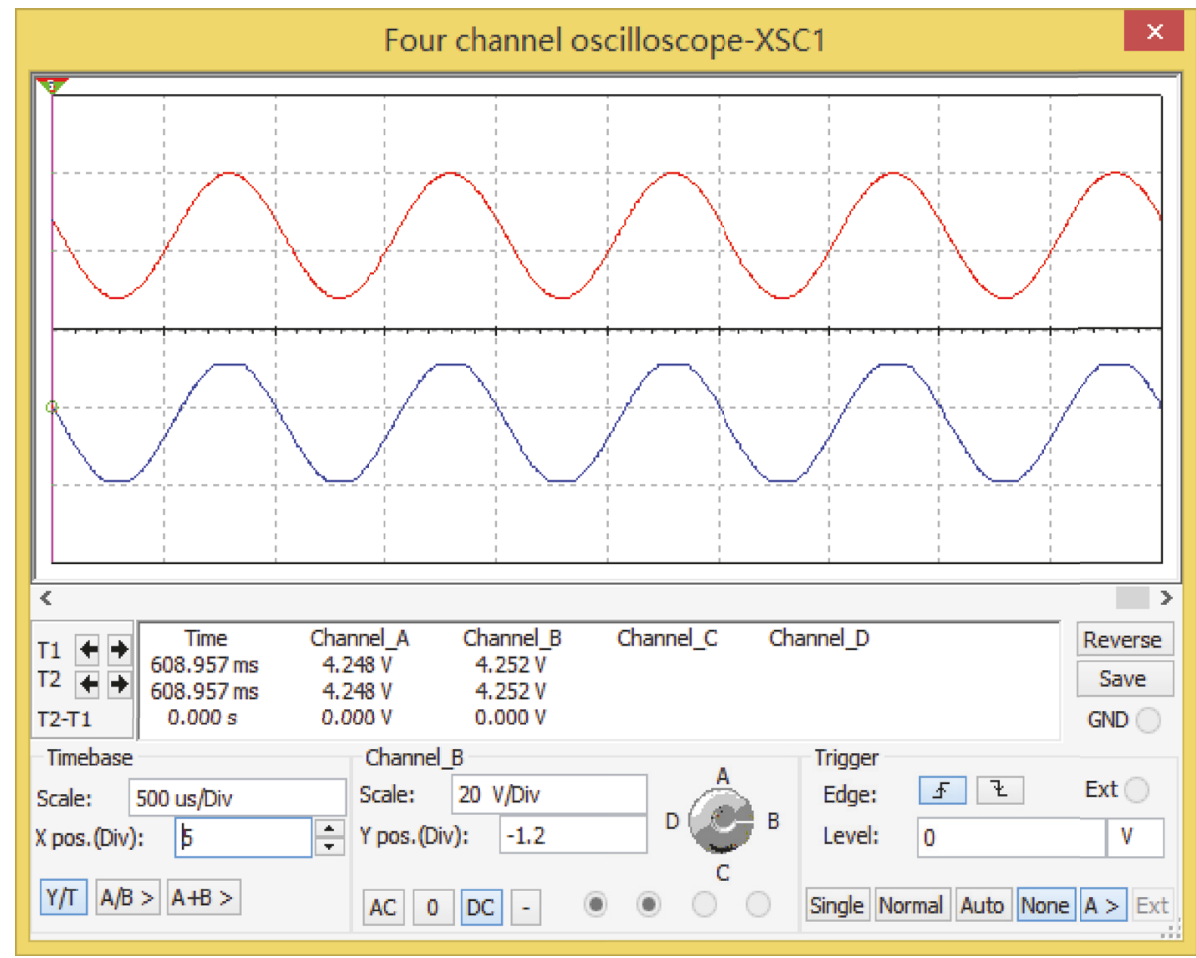

FiguRe 19: $16 \mathrm{~V}$.

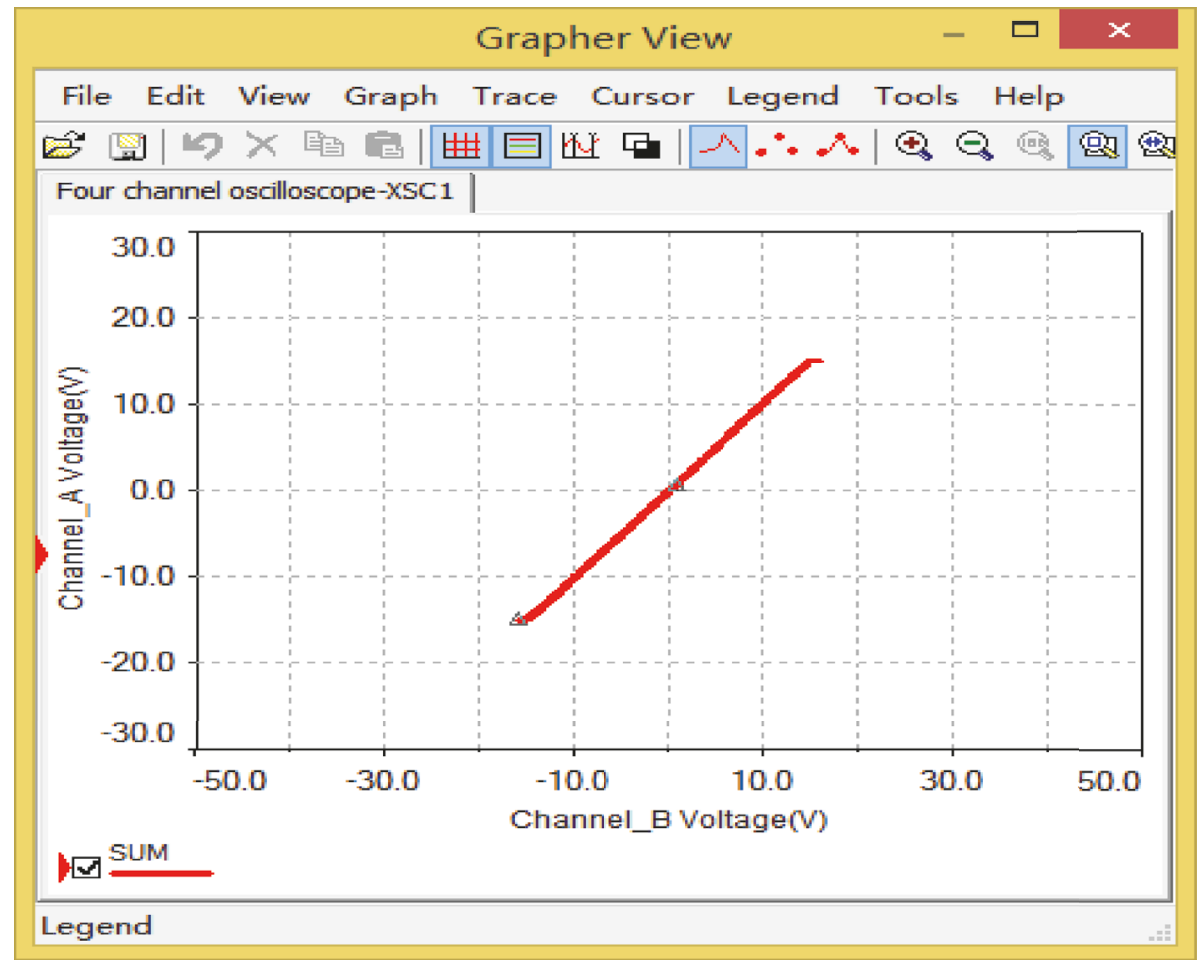

FIGURE 20: Synchronous phase portrait of $16 \mathrm{~V}$. 


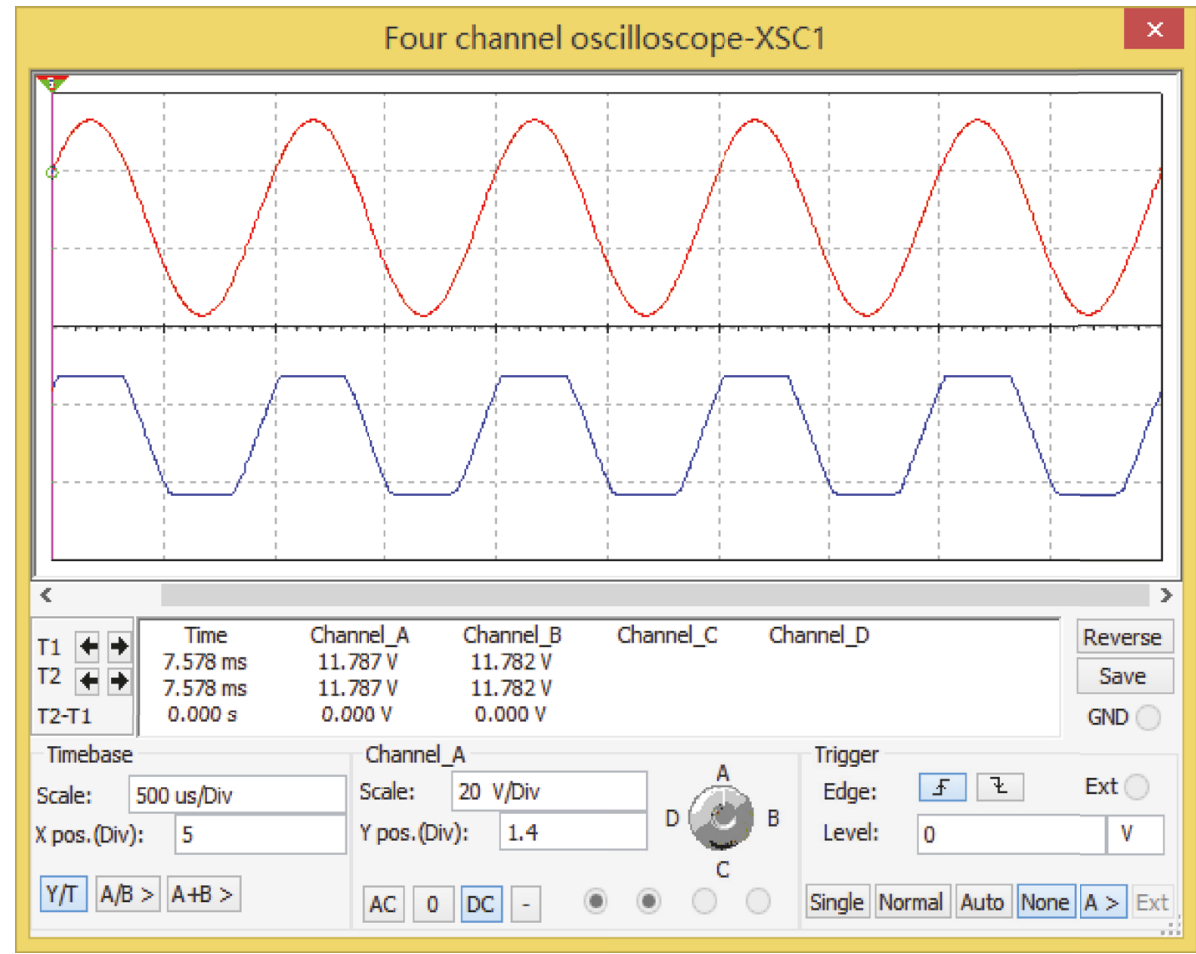

Figure 21: $25 \mathrm{~V}$.

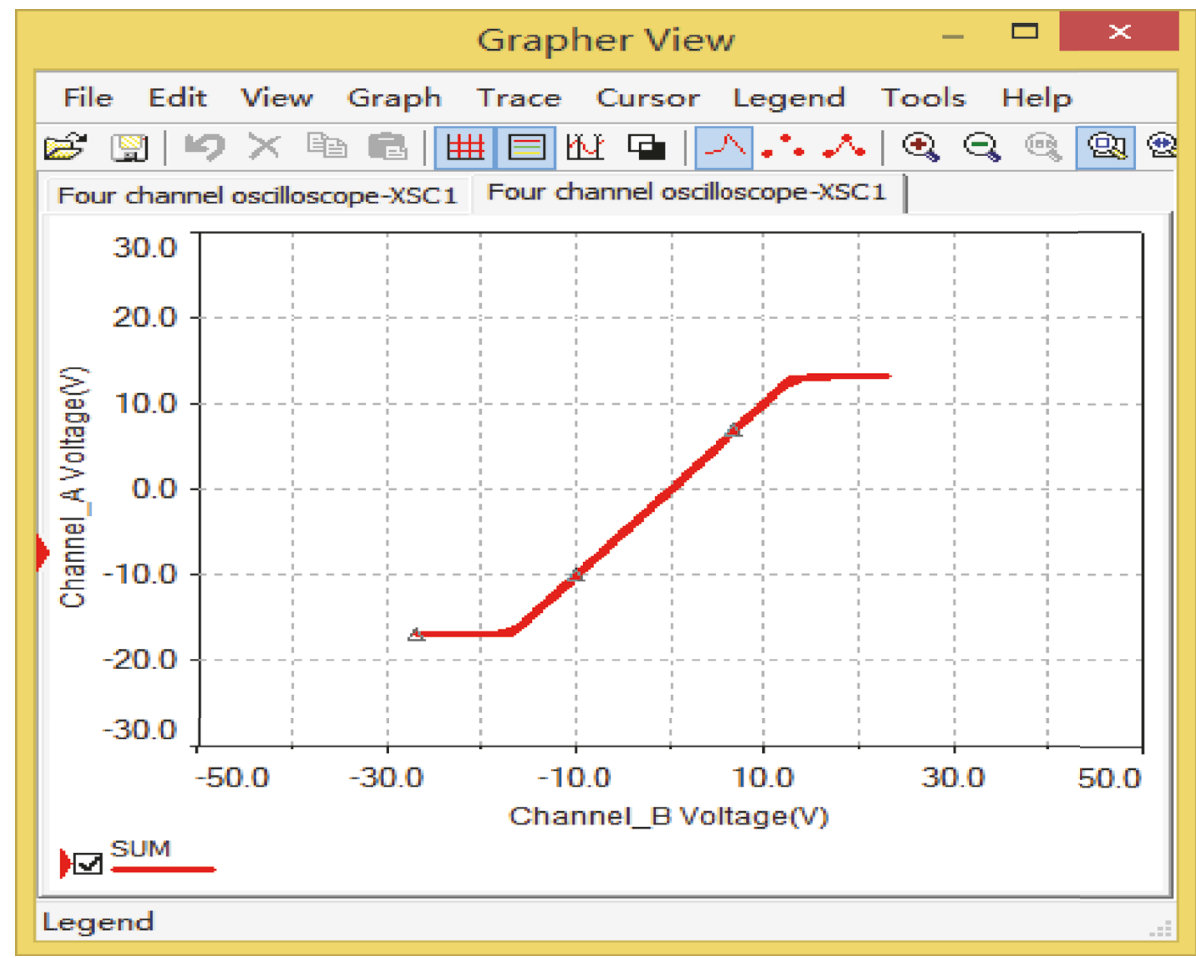

FIGURE 22: Synchronous phase portrait of $25 \mathrm{~V}$. 


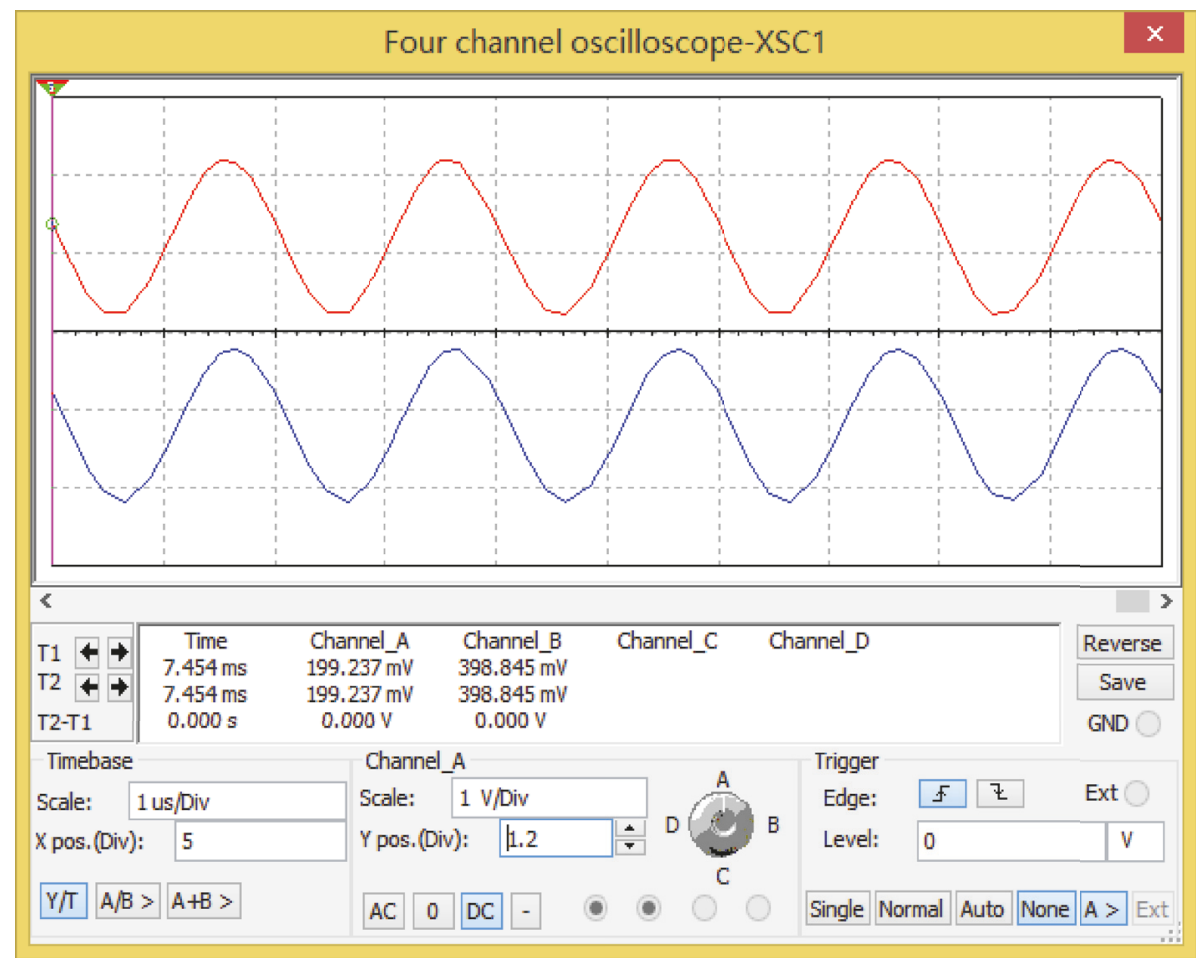

Figure 23: 500 KHZ.

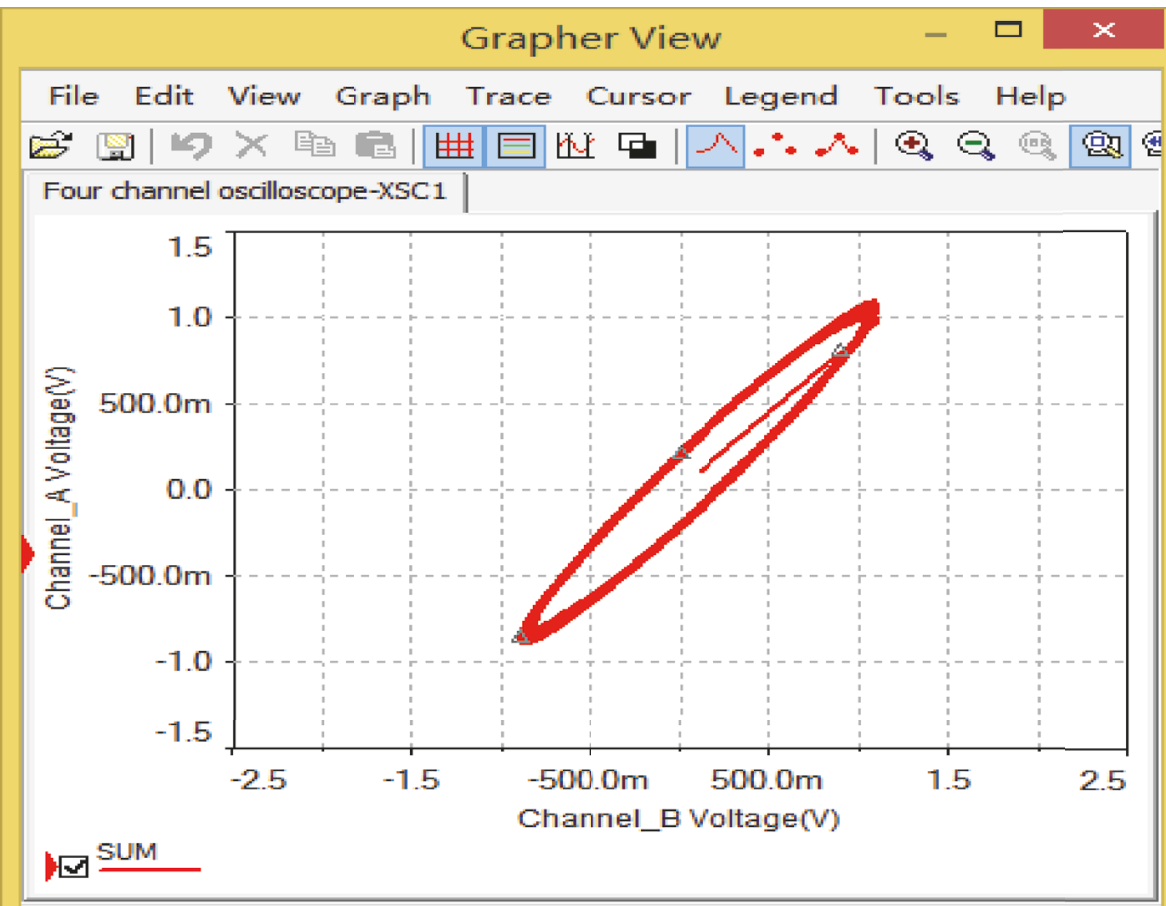

Selected Trace:SUM

FIGURE 24: Phase portrait of $500 \mathrm{KHZ}$. 


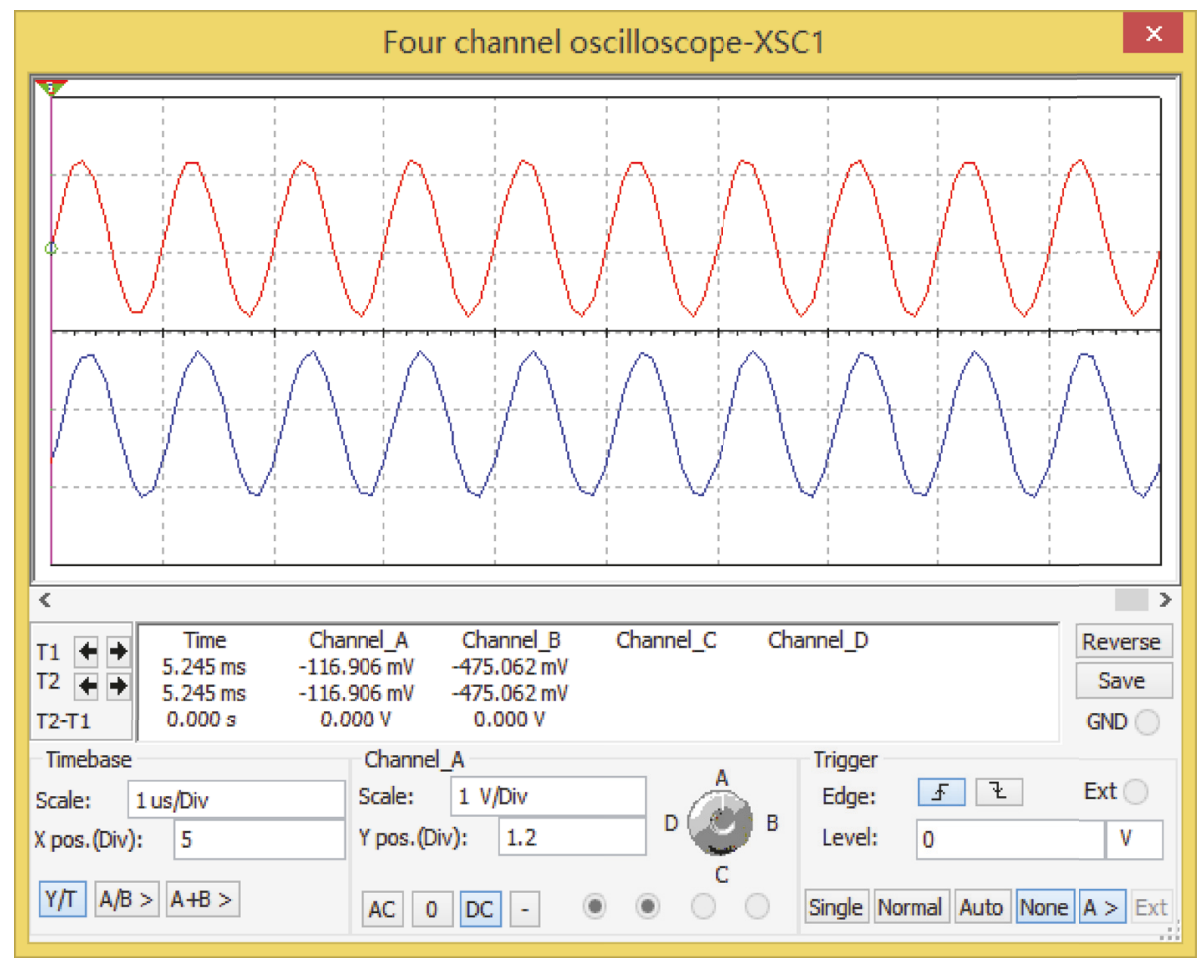

Figure 25: $1 \mathrm{MHZ}$.

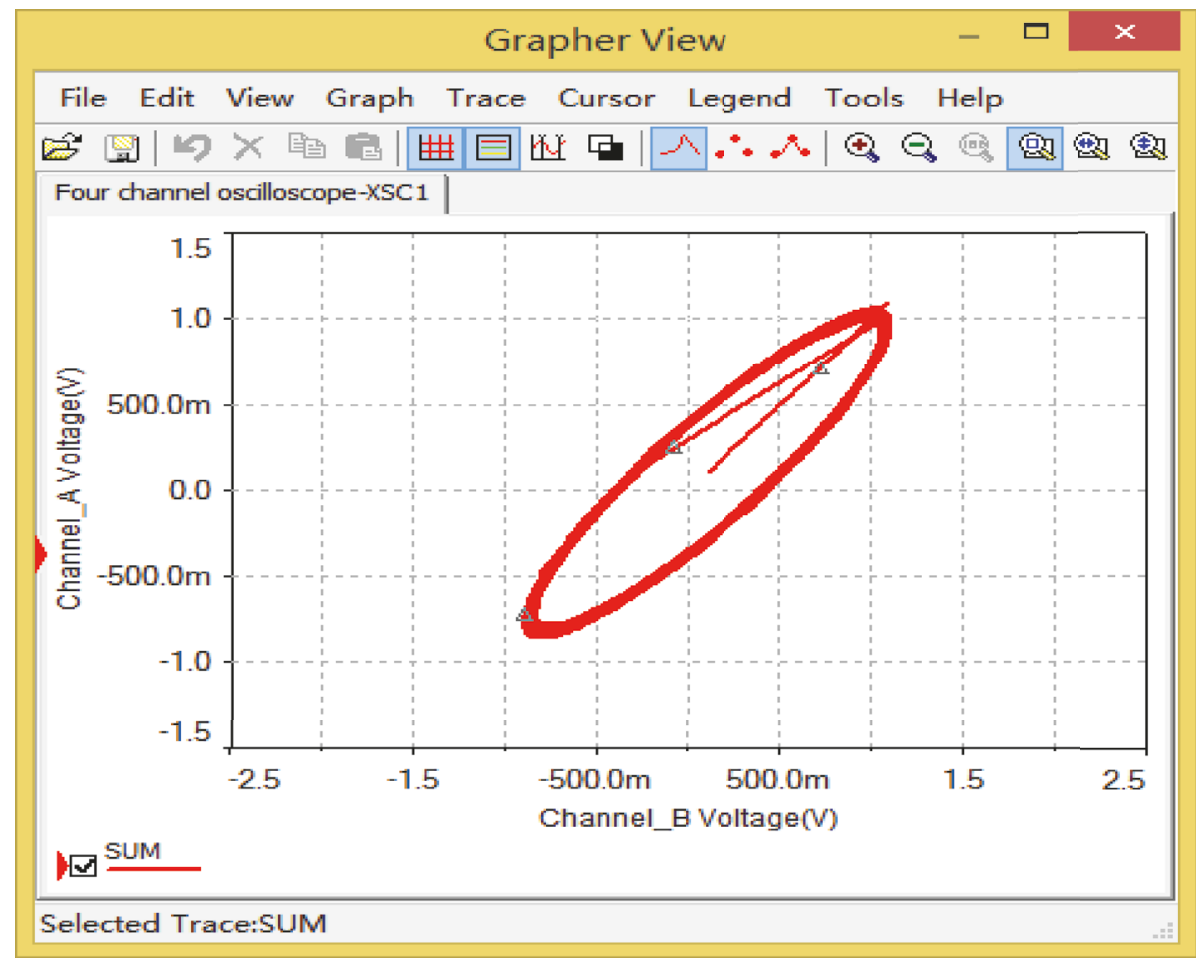

FIgURE 26: Phase portrait of $1 \mathrm{MHZ}$. 


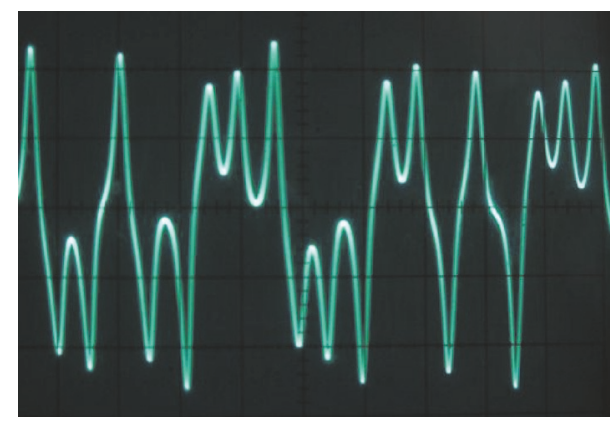

(a) $x$ waveform

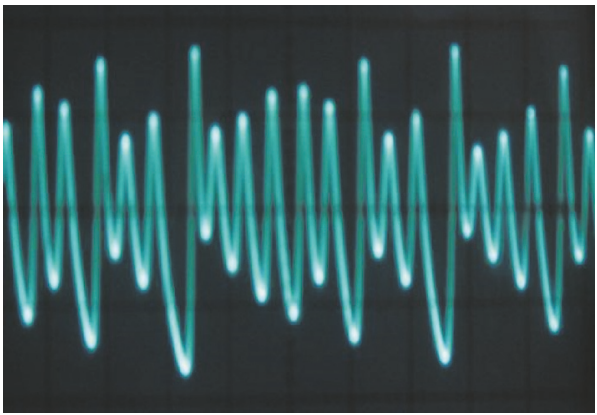

(c) $z$ waveform

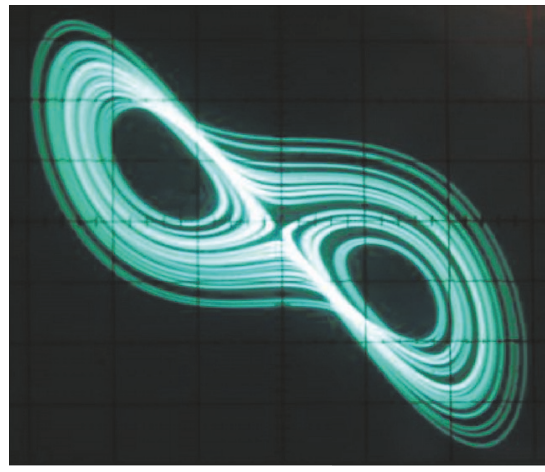

(e) $x y$ phase portrait

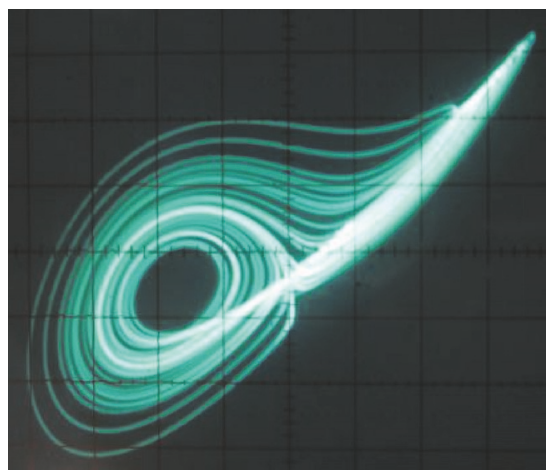

(h) $x u$ phase portrait

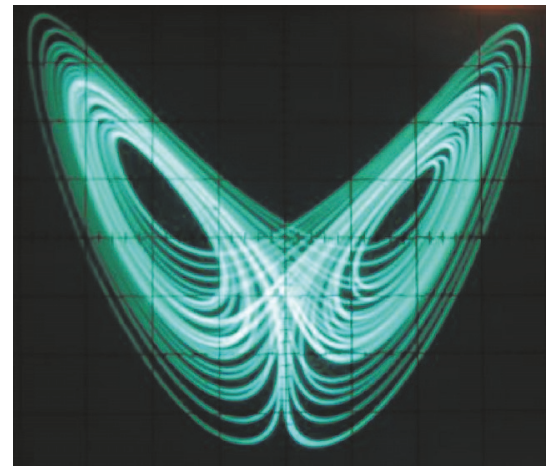

(f) $x z$ phase portrait

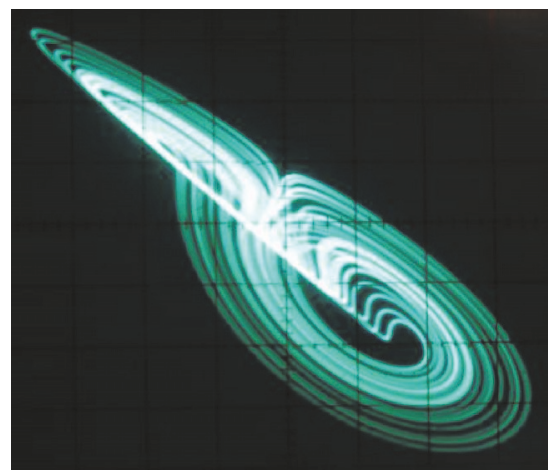

(i) $y u$ phase portrait

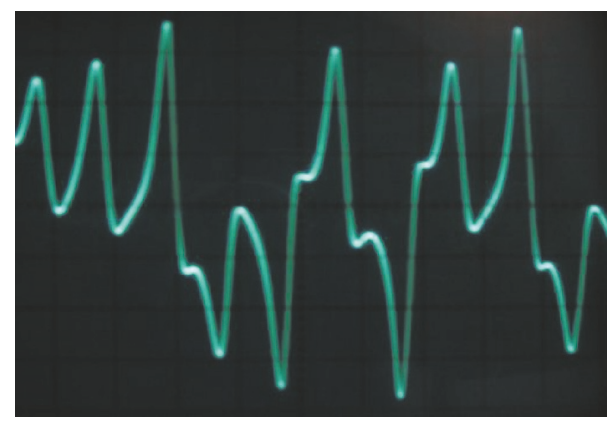

(b) $y$ waveform

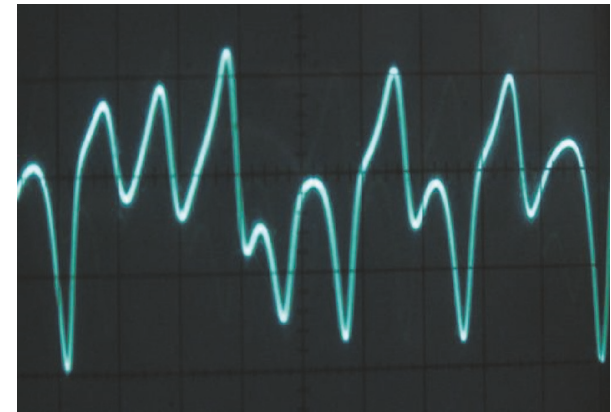

(d) $u$ waveform

FIGURE 27: The output waveforms and phase portraits signal photos of the novel $(y+z)-u-x$ hyperchaotic system. 


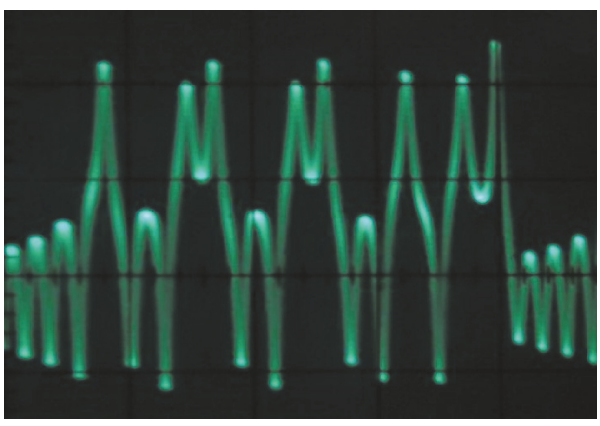

(a) $x$ waveform

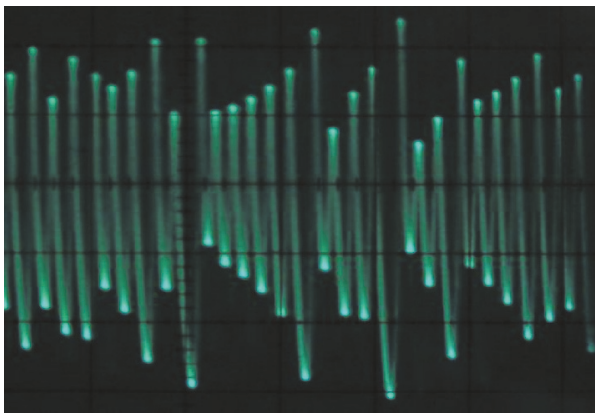

(c) $z$ waveform

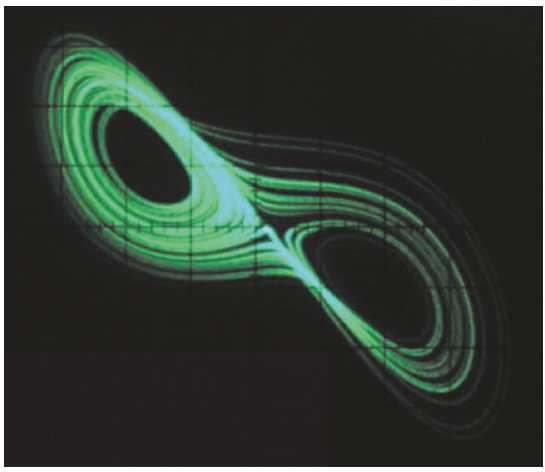

(e) $x y$ phase portrait

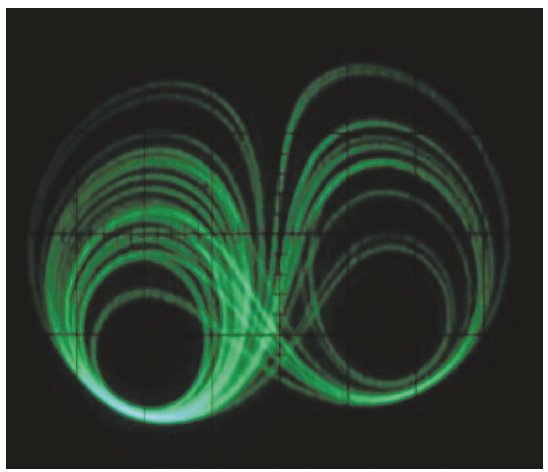

(h) $x u$ phase portrait

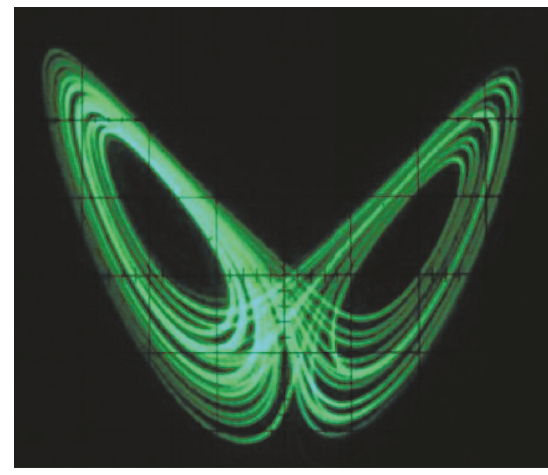

(f) $x z$ phase portrait

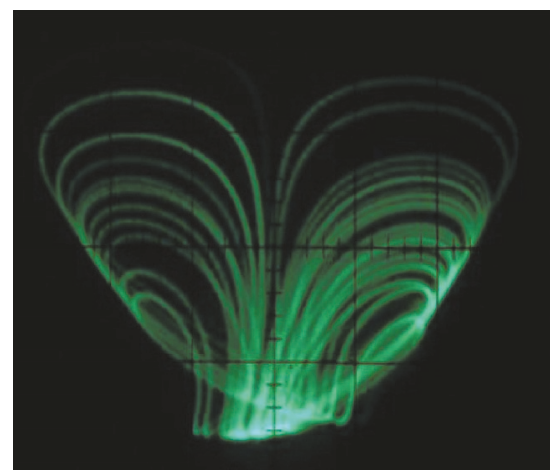

(i) $y u$ phase portrait

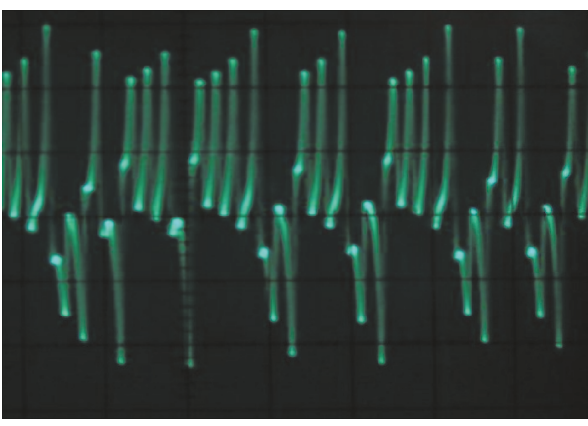

(b) $y$ waveform

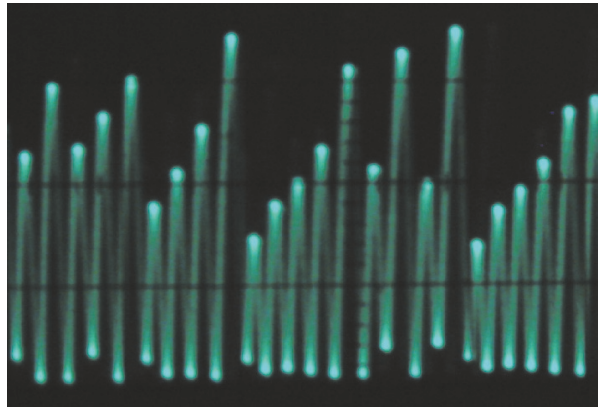

(d) $u$ waveform

FIGURE 28: The output waveforms and phase portraits signal photos of the novel $z-u-y$ hyperchaotic system. 
circuit has the following advantages: (i) higher intensity limit and stability of the transmitted signal, (ii) wider broadband characteristic than ordinary chaotic secure communication circuits, (iii) more stable working performance and smaller distortion, (iv) easier debugging and more convenience for mass production. Moreover, if three jumper pins are added, it can be used to control the new hyperchaotic secure communication circuit to realize the experiments of chaotic synchronization and chaotic nonsynchronization as well as experiments without signal and with signal. Similarly, the proposed chaotic secure communication method can also be applied to the $z-u-y$ hyperchaotic circuit and other complex hyperchaotic systems.

\section{Hardware Implementation of the Novel $(y+z)-u-x$ and $z-u-y$ Hyperchaotic Circuits}

Despite the fact that chaotic circuits have many advantages, the study of chaotic circuit is still in the phase of laboratory research. Because most researchers still concentrate on the study of chaos theory in numerical simulations, there is a certain deviation from the physical circuit system. In order to verify that the novel $(y+z)-u-x$ and $z-u-y$ hyperchaotic circuits have high accuracy and good robustness and further study the chaotic dynamical characteristics of the novel hyperchaotic systems (5) and (27), two practical electronic circuits are constructed using some general electronic components such as operational amplifiers, analog multipliers, resistors, and capacitors. Figures 27(a), 27(b), 27(c), and 27(d) show the output waveform photos of the novel $(y+z)-u-x$ hyperchaotic circuit. Figures 27(e), 27(f), 27(g), 27(h), 27(i), and $27(\mathrm{j})$ show the output phase portraits photos of the novel $(y+z)-u-x$ hyperchaotic circuit. Figures 28(a), 28(b), 28(c), and $28(\mathrm{~d})$ show the output waveform photos of the novel $z$-u- $y$ hyperchaotic circuit. Figures 28(e), 28(f), 28(g), 28(h), $28(\mathrm{i})$, and $28(\mathrm{j})$ show the output phase portraits photos of the novel $z-u-y$ hyperchaotic circuit.

It can be seen from the experimental results that the existence of the hyperchaotic attractors is proved, and it is also proved by the Multisim and Matlab simulation results. The proposed circuit design and circuit deformation methods of the novel fourth-order hyperchaotic system provide a reliable straightforward way of realizing chaotic circuits. The methods are easy to handle and prevent the output voltage from exceeding the limitation of the amplifier linear region efficiency.

\section{Conclusion}

In this paper, a novel hyperchaotic system is proposed based on a modified Lorenz-like chaotic circuit with reduced number of amplifiers. This paper is an attempt to investigate the dynamical behavior, synchronous stability, and applications in circuit deformation and secure communication field of the new hyperchaotic system. In order to enhance the confidentiality and security of the transmitted signals, the active control method is applied to achieve chaotic synchronization of the novel $(y+z)-u-x$ hyperchaotic system based on the Lyapunov stability theory. Comparisons between Multisim as well as Matlab simulation results and physical experimental results show that they are consistent with each other and demonstrate that an attractor of the hyperchaotic system exists. However, the chaotic secure communication method proposed in this paper still has some limitations. Since conventional amplifiers and current conveyors have frequency limitations, they have limited performance in the implementation of nonlinear circuits. And another important problem is how to improve the unpredictability of chaotic communication system. If the chaotic oscillator has higher positive Lyapunov exponents, those designs can be improved because it determines the unpredictability grade of chaotic oscillators.

That way, our future research will focus on the optimization of Lyapunov exponents and circuit implementation using embedded systems like FPGA, which can lead us to observe complex attractors with high Lyapunov exponent values.

\section{Conflicts of Interest}

The authors have declared that no conflicts of interest exist.

\section{Acknowledgments}

The authors are greatly thankful for the help and support from the Open Project of State Key Laboratory of ASIC \& System (Grant no. 2016KF004).

\section{References}

[1] E. N. Lorenz, "Deterministic nonperiodic flow," Journal of the Atmospheric Sciences, vol. 20, no. 2, pp. 130-141, 1963.

[2] K. M. Cuomo and A. V. Oppenheim, "Circuit implementation of synchronized chaos with applications to communications," Physical Review Letters, vol. 71, no. 1, pp. 65-68, 1993.

[3] L.-H. Yu and J.-C. Fang, "Synchronization of chaotic neural networks based on adaptive inverse control and its applications in secure communications," Acta Physica Sinica, vol. 54, no. 9, pp. 4012-4018, 2005.

[4] B. Muthuswamy and L. O. Chua, "Simplest chaotic circuit," International Journal of Bifurcation and Chaos, vol. 20, no. 5, pp. 1567-1580, 2010.

[5] Y. Liu and Q. Yang, "Dynamics of a new Lorenz-like chaotic system," Nonlinear Analysis: Real World Applications, vol. 11, no. 4, pp. 2563-2572, 2010.

[6] X. G. Zhang, H. T. Sun, J. L. Zhao et al., "Equivalent circuit in function and topology to Chuas circuit and the design methods of these circuits," Acta Physica Sinica, vol. 63, no. 20, pp. 1-8, 2014.

[7] G. A. Leonov and N. V. Kuznetsov, "On differences and similarities in the analysis of Lorenz, Chen, and LU systems," Applied Mathematics and Computation, vol. 256, pp. 334-343, 2015.

[8] G. M. Mahmoud, M. E. Ahmed, and E. E. Mahmoud, "Analysis of hyperchaotic complex Lorenz systems," International Journal of Modern Physics C, vol. 19, no. 10, pp. 1477-1494, 2008.

[9] G. M. Mahmoud and E. E. Mahmoud, "Synchronization and control of hyperchaotic complex Lorenz system," Mathematics 
and Computers in Simulation, vol. 80, no. 12, pp. 2286-2296, 2010.

[10] A. M. El-Sayed, H. M. Nour, A. Elsaid, A. E. Matouk, and A. Elsonbaty, "Circuit realization, bifurcations, chaos and hyperchaos in a new 4D system," Applied Mathematics and Computation, vol. 239, pp. 333-345, 2014.

[11] L. Merah, A. Ali-Pacha, N. H. Said, and M. Mamat, "Design and FPGA implementation of Lorenz chaotic system for information security issues," Applied Mathematical Sciences, vol. 7, no. 5-8, pp. 237-246, 2013.

[12] M. Halimi, K. Kemih, and M. Ghanes, "Circuit simulation of an analog secure communication based on synchronized Chaotic Chua's system," Applied Mathematics \& Information Sciences, vol. 8, no. 4, pp. 1509-1516, 2014.

[13] M. Zapateiro, Y. Vidal, and L. Acho, "A secure communication scheme based on chaotic Duffing oscillators and frequency estimation for the transmission of binary-coded messages," Communications in Nonlinear Science and Numerical Simulation, vol. 19, no. 4, pp. 991-1003, 2014.

[14] E. Tlelo-Cuautle, V. H. Carbajal-Gomez, P. J. Obeso-Rodelo, J. J. Rangel-Magdaleno, and J. C. Núñez-Pérez, "FPGA realization of a chaotic communication system applied to image processing," Nonlinear Dynamics, vol. 82, no. 4, pp. 1879-1892, 2015.

[15] T.-C. Lin, F.-Y. Huang, Z. Du, and Y.-C. Lin, "Synchronization of fuzzy modeling chaotic time delay memristor-based Chua's circuits with application to secure communication," International Journal of Fuzzy Systems, vol. 17, no. 2, pp. 206-214, 2015.

[16] H.-C. Chen, B.-Y. Liau, and Y.-Y. Hou, "Hardware implementation of lorenz circuit systems for secure chaotic communication applications," Sensors, vol. 13, no. 2, pp. 2494-2505, 2013.

[17] W. Shen, Z. Zeng, and L. Wang, "Stability analysis for uncertain switched neural networks with time-varying delay," Neural Networks, vol. 83, pp. 32-41, 2016.

[18] Y. Cao, S. Wen, M. Z. Q. Chen, T. Huang, and Z. Zeng, "New results on anti-synchronization of switched neural networks with time-varying delays and lag signals," Neural Networks, vol. 81, pp. 52-58, 2016.

[19] C. Feng, L. Cai, Q. Kang, S. Wang, and H. Zhang, "Novel hyperchaotic system and its circuit implementation," Journal of Computational and Nonlinear Dynamics, vol. 10, no. 6, Article ID 4029227, 2015.

[20] E. E. Mahmoud, "Complex complete synchronization of two nonidentical hyperchaotic complex nonlinear systems," Mathematical Methods in the Applied Sciences, vol. 37, no. 3, pp. 321328, 2014.

[21] E. E. Mahmoud, "Dynamics and synchronization of new hyperchaotic complex Lorenz system," Mathematical and Computer Modelling, vol. 55, no. 7-8, pp. 1951-1962, 2012.

[22] G. M. Mahmoud and E. E. Mahmoud, "Complete synchronization of chaotic complex nonlinear systems with uncertain parameters," Nonlinear Dynamics, vol. 62, no. 4, pp. 875-882, 2010.

[23] V. Sundarapandian and I. Pehlivan, "Analysis, control, synchronization, and circuit design of a novel chaotic system," Mathematical and Computer Modelling, vol. 55, no. 7-8, pp. 1904-1915, 2012.

[24] I. Pehlivan, I. M. Moroz, and S. Vaidyanathan, "Analysis, synchronization and circuit design of a novel butterfly attractor," Journal of Sound and Vibration, vol. 333, no. 20, pp. 5077-5096, 2014.
[25] L. Wang, Z. Zeng, J. Hu, and X. Wang, "Controller design for global fixed-time synchronization of delayed neural networks with discontinuous activations," Neural Networks, vol. 87, pp. 122-131, 2017.

[26] P. S. Swathy and K. Thamilmaran, "Hyperchaos in SC-CNN based modified canonical Chua's circuit," Nonlinear Dynamics, vol. 78, no. 4, pp. 2639-2650, 2014.

[27] G. A. Leonov, N. V. Kuznetsov, and T. N. Mokaev, "Hidden attractor and homoclinic orbit in Lorenz-like system describing convective fluid motion in rotating cavity," Communications in Nonlinear Science and Numerical Simulation, vol. 28, no. 1-3, pp. 166-174, 2015.

[28] J. Ma, F. Wu, G. Ren, and J. Tang, "A class of initials-dependent dynamical systems," Applied Mathematics and Computation, vol. 298, pp. 65-76, 2017.

[29] R. Trejo-Guerra, E. Tlelo-Cuautle, J. Jimenez-Fuentes et al., "Integrated circuit generating 3- and 5-scroll attractors," Communications in Nonlinear Science and Numerical Simulation, vol. 17, no. 11, pp. 4328-4335, 2012.

[30] F. Li and C. Yao, "The infinite-scroll attractor and energy transition in chaotic circuit," Nonlinear Dynamics, vol. 84, no. 4, pp. 2305-2315, 2016.

[31] D. A. Prousalis, C. K. Volos, I. N. Stouboulos, and I. M. Kyprianidis, "A novel 4-D hyperchaotic four-wing memristive system," in Proceedings of the 5th International Conference on Modern Circuits and Systems Technologies, MOCAST 2016, grc, May 2016.

[32] J. M. Muñoz-Pacheco, E. Tlelo-Cuautle, I. Toxqui-Toxqui, C. Sánchez-López, and R. Trejo-Guerra, "Frequency limitations in generating multi-scroll chaotic attractors using CFOAs," International Journal of Electronics, vol.101, no. 11, pp. 1559-1569, 2014.

[33] R. L. Filali, M. Benrejeb, and P. Borne, “On observer-based secure communication design using discrete-time hyperchaotic systems," Communications in Nonlinear Science and Numerical Simulation, vol. 19, no. 5, pp. 1424-1432, 2014.

[34] X. Li, Y.-J. Lu, Y.-F. Zhang, X.-G. Zhang, and P. Gupta, "Design and hardware implementation of a new chaotic secure communication technique," PLoS ONE, vol. 11, no. 8, Article ID e0158348, 2016. 


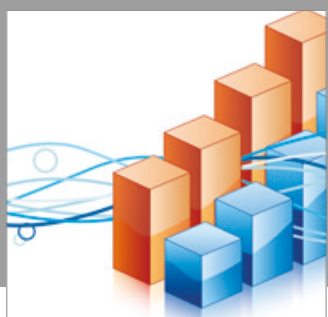

Advances in

Operations Research

vatersals

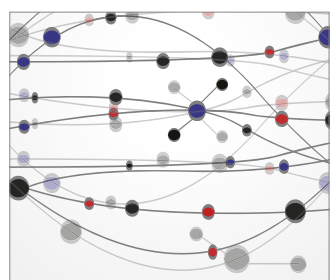

\section{The Scientific} World Journal
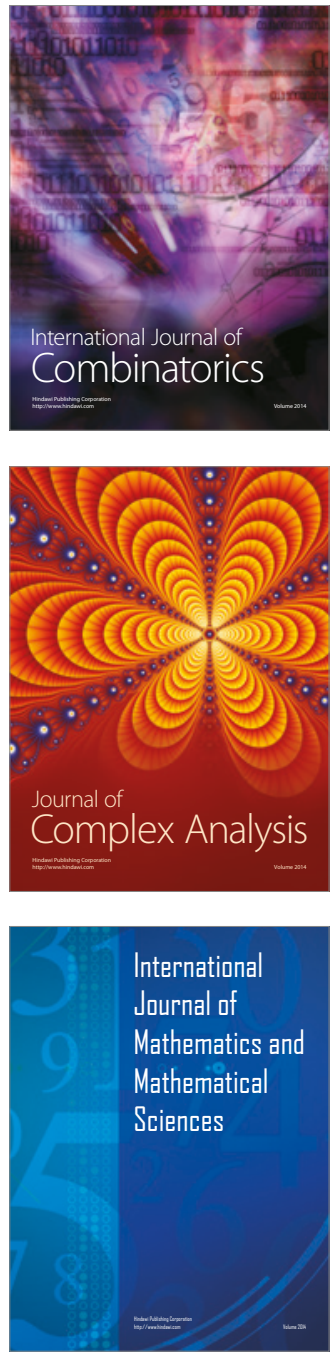
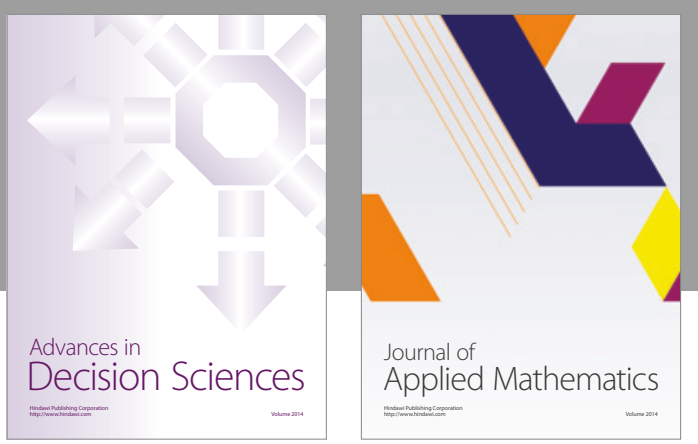

Algebra

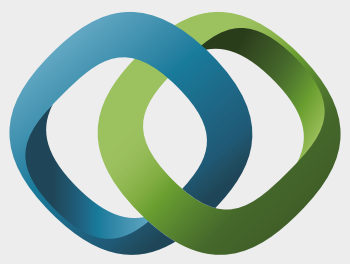

\section{Hindawi}

Submit your manuscripts at

https://www.hindawi.com
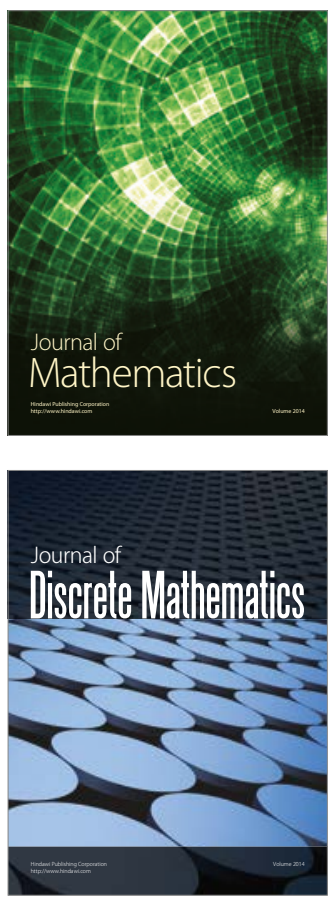

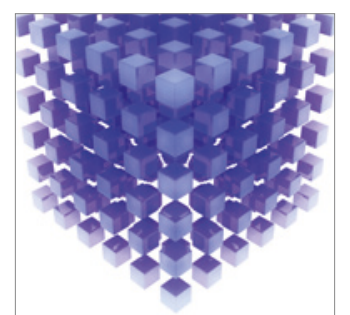

Mathematical Problems in Engineering
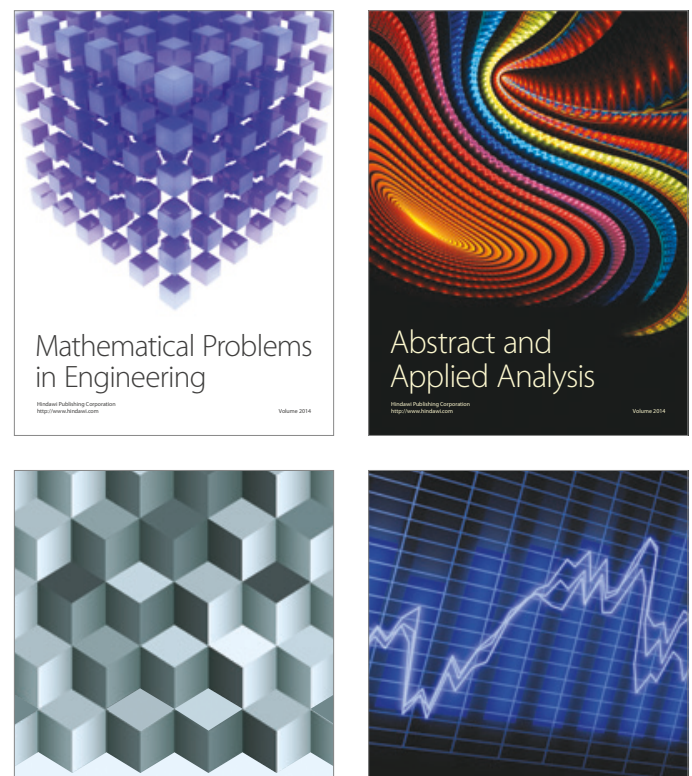

Journal of

Function Spaces

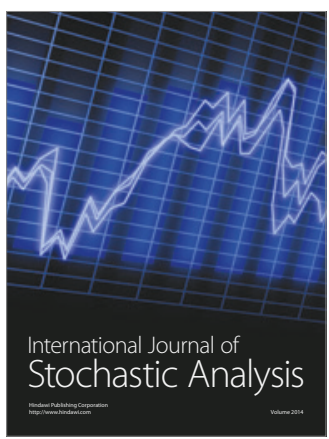

Probability and Statistics
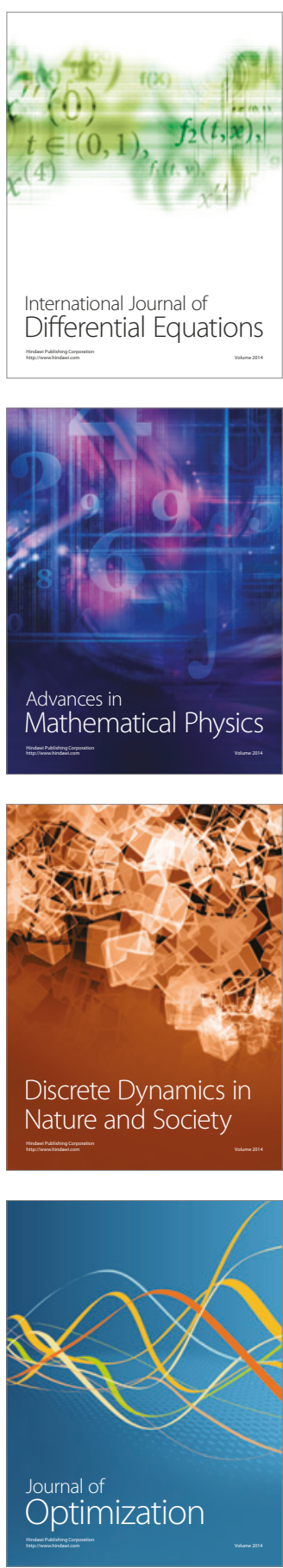